\title{
Investigating the Origins of Two Extreme Solar Particle Events: Proton Source Profile and Associated Electromagnetic Emissions
}

\author{
Leon Kocharov $^{1}$, Silja Pohjolainen ${ }^{2}$, Alexander Mishev ${ }^{3}$, Mike J. Reiner ${ }^{4}$, Jeongwoo Lee ${ }^{5,6}$, Timo Laitinen ${ }^{7}$, Leonid V. Didkovsky ${ }^{8}$, \\ Victor J. Pizzo ${ }^{9}$, Roksoon Kim ${ }^{10}$, Andreas Klassen ${ }^{11}$, Marian Karlicky ${ }^{12}$, Kyung-Suk Cho ${ }^{10,13}$, Dale E. Gary ${ }^{6}$, Ilya Usoskin ${ }^{1,3}$, \\ Eino Valtonen ${ }^{14}$, and Rami Vainio ${ }^{14}$ \\ ${ }^{1}$ Sodankylä Geophysical Observatory/Oulu Unit, University of Oulu, P.O.B. 3000, Oulu FI-90014, Finland \\ ${ }^{2}$ Tuorla Observatory, University of Turku, Piikkiö FI-21500, Finland \\ ${ }^{3}$ Space Climate Research Unit, University of Oulu, Oulu FI-90014, Finland \\ ${ }^{4}$ The Catholic University of America, Washington, DC, and NASA/Goddard Space Flight Center, Greenbelt, MD, USA \\ ${ }^{5}$ Department of Physics and Astronomy, Seoul National University, Seoul 151-747, Korea \\ ${ }^{6}$ Center for Solar-Terrestrial Research, New Jersey Institute of Technology, Newark NJ 07102-1982, USA \\ 7 Jeremiah Horrocks Institute, University of Central Lancashire, Preston PR1 2HE, UK \\ ${ }^{8}$ University of Southern California Space Sciences Center, 835 Bloom Walk, Los Angeles CA 90089, USA \\ ${ }^{9}$ NOAA Space Weather Prediction Center, Boulder, CO 80305, USA \\ ${ }^{10}$ Korea Astronomy and Space Science Institute, Daejeon 305-348, Korea \\ ${ }^{11}$ Institut für Experimentelle und Angewandte Physik, Christian-Albrechts-Universität, Kiel D-24118, Germany \\ ${ }^{12}$ Astronomical Institute of the Czech Academy of Sciences, Fričova 258, Ondřejov 251 65, Czech Republic \\ ${ }^{13}$ University of Science and Technology, Daejeon 305-348, Korea \\ ${ }^{14}$ Space Research Laboratory, University of Turku, Turku FI-20014, Finland \\ Received 2016 October 24; revised 2017 March 27; accepted 2017 March 27; published 2017 April 18
}

\begin{abstract}
We analyze the high-energy particle emission from the Sun in two extreme solar particle events in which protons are accelerated to relativistic energies and can cause a significant signal even in the ground-based particle detectors. Analysis of a relativistic proton event is based on modeling of the particle transport and interaction, from a nearSun source through the solar wind and the Earth's magnetosphere and atmosphere to a detector on the ground. This allows us to deduce the time profile of the proton source at the Sun and compare it with observed electromagnetic emissions. The 1998 May 2 event is associated with a flare and a coronal mass ejection (CME), which were well observed by the Nançay Radioheliograph, thus the images of the radio sources are available. For the 2003 November 2 event, the low corona images of the CME liftoff obtained at the Mauna Loa Solar Observatory are available. Those complementary data sets are analyzed jointly with the broadband dynamic radio spectra, EUV images, and other data available for both events. We find a common scenario for both eruptions, including the flare's dual impulsive phase, the CME-launch-associated decimetric-continuum burst, and the late, low-frequency type III radio bursts at the time of the relativistic proton injection into the interplanetary medium. The analysis supports the idea that the two considered events start with emission of relativistic protons previously accelerated during the flare and $\mathrm{CME}$ launch, then trapped in large-scale magnetic loops and later released by the expanding CME.
\end{abstract}

Key words: acceleration of particles - shock waves - Sun: coronal mass ejections (CME) - Sun: particle emission

\section{Introduction}

A major solar eruption comprises both a gradual solar flare, observed in X-rays and other electromagnetic emissions, and a wide and fast coronal mass ejection (CME). There are evidences that the high-energy $(\gtrsim 50 \mathrm{MeV})$ protons can be produced by both flare and CME, while relative contributions of the eruption stages to particles arriving at 1 au are still under discussion (e.g., Gopalswamy et al. 2012). Detection of solar protons and $\alpha$-particles above $\sim 0.4 \mathrm{GeV} \mathrm{nucl}^{-1}$ in the strongest events is possible with ground-level detectors, like neutron monitors (NMs), which historically preceded the particle measurements in space. The ground level enhancement (GLE) events are considered extreme particle events that occur only about a dozen times during a solar cycle (e.g., Gopalswamy et al. 2016). The GLEs are rare but have been continuously studied for more than half a century (for a review see Ryan et al. 2000 and Shea \& Smart 2012).

Modern spaceborne instruments, like the Payload for Antimatter Matter Exploration and Light-nuclei Astrophysics (Adriani et al. 2011) and the Alpha Magnetic Spectrometer
(AMS; Ting 2013) can measure high-energy nuclei with a good resolution in both energy and arrival direction, but due to their low orbits, they suffer regular gaps in the solar particle observations. Continuous and accurate measurements are possible on board the Solar and Heliospheric Observatory (SOHO) spacecraft orbiting around the Lagrange point L1, but particle instruments on $\mathrm{SOHO}$ originally were not aimed at detecting protons and helium with energy above $120 \mathrm{MeV}$ nucl $^{-1}$ (Torsti et al. 1995). Hence, one has to combine together the particle data of different detectors.

Along with particle detectors, $\mathrm{SOHO}$ carries a set of solar telescopes and coronagraphs, and 20 years of $\mathrm{SOHO}$ operations have made multi-wavelength investigations a common practice in the solar energetic particle (SEP) research. It is common to compare the timing of the "first particle" emission from the Sun with observed electromagnetic emissions, but such consideration may be not sufficient, because in the major (gradual) SEP events, the particle injection from the Sun is a prolonged process, so that the entire time profile of the SEP source should be considered. 


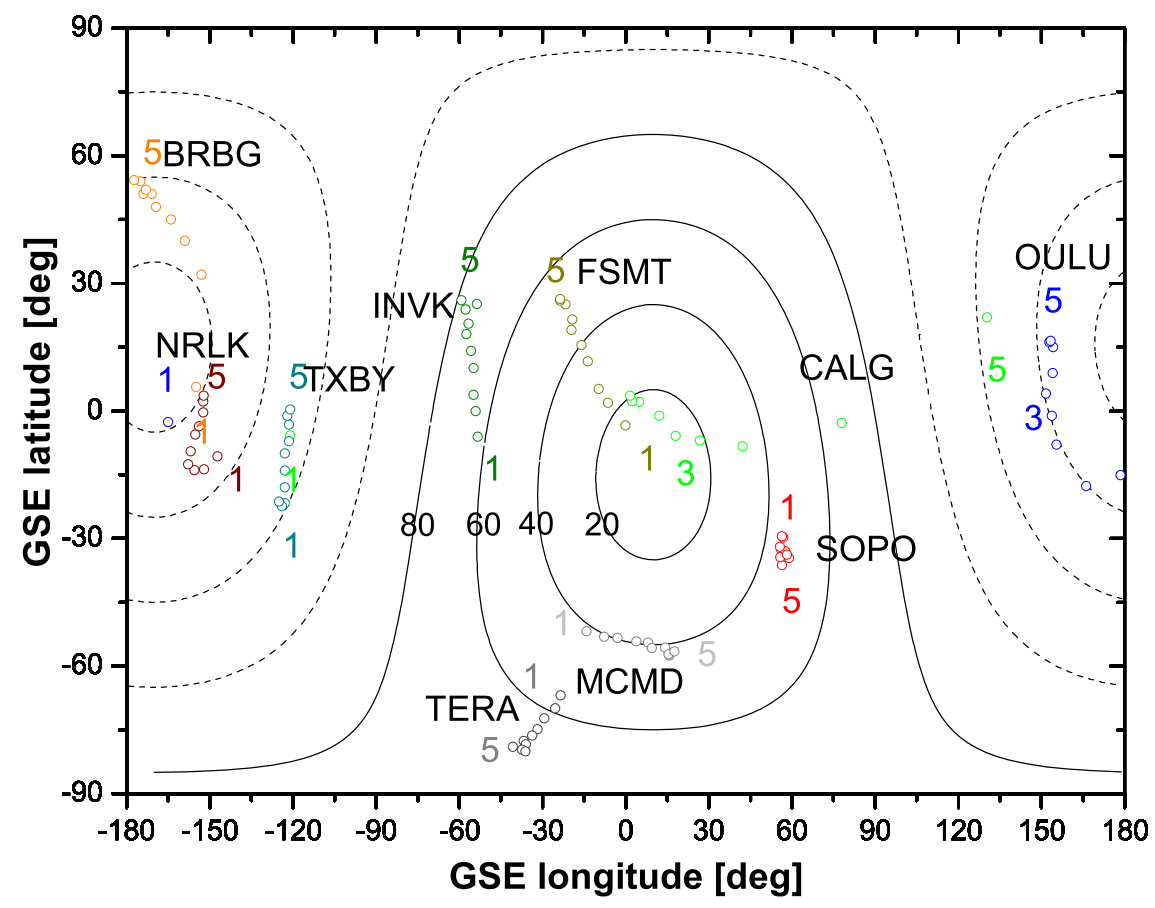

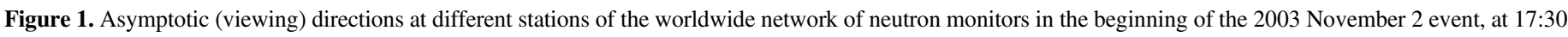

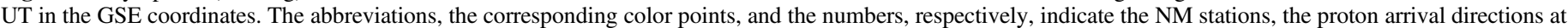

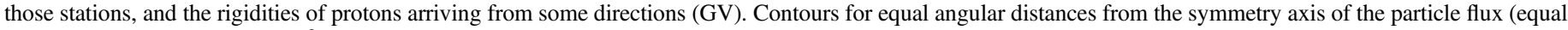

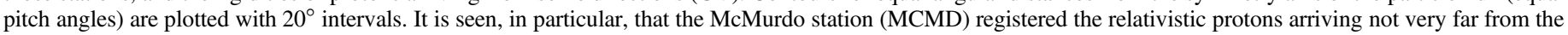
flux axis, at the pitch angles $\alpha \approx 40^{\circ}$, while the Oulu neutron monitor detected protons arriving from almost anti-Sun direction $\alpha \approx 140^{\circ}-160^{\circ}$.

For the present study, we have selected two apparently similar GLEs: the 1998 May 2 event (GLE 56) and the 2003 November 2 event (GLE 67). We start with an analysis of particle data and particle transport modeling, from the Sun to a detector on the ground, in order to deduce the particle source profile at the Sun. Then, the inferred source profile is compared to the multi-wavelength data of solar observations, and possible solar origins of the extreme particle events are investigated.

\section{Instrumentation and Data}

Spectral and angular characteristics of solar protons of highest energies, $\sim 0.4-10 \mathrm{GeV}$, can be inferred from the records of the worldwide network of ground-based neutron monitors, if a relationship between the NM count rates and the primary particles in the interplanetary space is established. That requires a modeling of particle transport and interaction in the Earth's magnetosphere and atmosphere (Debrunner \& Brunberg 1968; Hatton 1971; Debrunner \& Lockwood 1980; Shea \& Smart 1982; Cramp et al. 1997; Clem \& Dorman 2000; Dorman 2004; Desorgher et al. 2009; Mishev \& Usoskin 2013). In this study, we use the GLE data from the database stored in Oulu at http://gle.oulu.fi and the newly computed $\mathrm{NM}$ yield function that explicitly considers the lateral extent of the cosmic-ray-induced nuclear cascade in the atmosphere and provides a good agreement with experimental latitude surveys and other measurements (Mishev et al. 2013; Gil et al. 2015).

Analysis of a GLE using the NM data consists of several consecutive steps: a determination for each NM station of the rigidity-dependent asymptotic (outside magnetosphere) viewing directions and the rigidity cut-off by modeling of particle propagation in the magnetosphere; an initial guess of the inverse problem, either by assuming that the apparent source position is located near the direction of the interplanetary magnetic field (IMF) or using a procedure proposed by Cramp et al. (1995); and finally, the application of an optimization procedure (inverse method) for the derivation of the primary particle energy spectrum, the direction of the particle flux axis, and the particle pitch angle distribution. More details on the employed techniques are given in the Appendices. Figure 1 exemplifies a result of the viewing direction computation for the beginning of the 2003 November 2 event (GLE 67). It is seen that different NM stations are sensitive to primary protons arriving from different asymptotic directions, and thus the entire network should be used as a single instrument for the particle flux anisotropy measurements, still with a limited energy resolution.

Differential particle measurements in narrow energy channels with a good resolution in the particle species and arrival directions are possible with designated instruments on board spacecraft orbiting outside the magnetosphere. Such spaceborne measurements are available from the High Energy Detector of the ERNE instrument on board $\mathrm{SOHO}$ in the energy range of $11-120 \mathrm{MeV} \mathrm{nucl}^{-1}$ (Torsti et al. 1995). In the hecto-MeV range, the GOES data are available, but they do not allow particle flux anisotropy measurements, which are crucial for estimating the particle mean-free path and transport time from Sun to Earth.

Transport of SEPs from the near-Sun source to a detector at/ near the Earth may proceed in different structures of the IMF. We acquire the IMF data from in situ measurements on board the Advanced Composition Explorer (ACE) spacecraft, which, like $\mathrm{SOHO}$, orbits around the Lagrange point L1. The magnetic field is measured by the Magnetometer instrument (Smith 1998) and the solar wind plasma measurements are done with the Solar Wind Electron, Proton, and Alpha Monitor (McComas 1998). The data are provided by the ACE Science Center 
(http://www.srl.caltech.edu/ACE/ASC/; Appendix A). We also use the in situ plasma data from the Proton Monitor of the Charge, Element, and Isotope Analysis System on SOHO (Ipavich et al. 1998).

For analyses of the GLE-producing solar eruptions, we consider multi-wavelength data from $\mathrm{SOHO}$ telescopes and other instruments in space and on the ground. We extensively employ the solar EUV full-disk images in the $19.5 \mathrm{~nm}$ passband from the SOHO Extreme ultraviolet Imaging Telescope (EIT; Delaboudiniere et al. 1995). For the differential images, a pair of images with the highest cadence of about 12 minutes is typically used.

The CME height at the GLE onset time is typically about $2 R_{\odot}-3 R_{\odot}$ (Gopalswamy et al. 2012). For this reason, images of the low and middle corona are especially important. For the height range of $\sim 2.25 R_{\odot}-7 R_{\odot}$, the data are available from the C2 coronagraph of the Large Angle and Spectrometric COronagraph (LASCO) instrument on SOHO, and above $\sim 3.7 R_{\odot}$ from LASCO C3 (Brueckner et al. 1995). We use data from the LASCO CME catalog generated and maintained at the CDAW Data Center by NASA and The Catholic University of America in cooperation with the Naval Research Laboratory, from http://cdaw.gsfc.nasa.gov (Yashiro et al. 2004). In a few of the figures in the present paper, we also show the position of the CME leading edge defined with the Solar Eruptive Event Detection System of George Mason University (GMU SEEDS) from http://spaceweather.gmu.edu/seeds/. In such figures, the blue color on the difference image indicates the position of the leading edge, while the red color indicates an approximate outline to the leading edge that was created using a segmentation technique (Olmedo et al. 2008).

In the case of the 2003 November 2 event, also available are the low corona images from the $\mathrm{Mk} 4$ coronameter of the Mauna Loa Solar Observatory (MLSO; http://www2.hao.ucar.edu; Elmore et al. 2003) operated by the High Altitude Observatory, as part of the National Center for Atmospheric Research (NCAR supported by the National Science Foundation). We outline the position of the CME leading edge as seen in the difference images with the cadence of 3 minutes.

The meter-wavelength radio images were acquired by the Nançay radioheliograph $(\mathrm{NRH})$ in France. It operates at five frequencies 164, 236, 327, 410, and $432 \mathrm{MHz}$ with a spatial resolution of $\sim 3^{\prime}$ at $164 \mathrm{MHz}$ and of $\sim 1^{\prime}$ at $432 \mathrm{MHz}$ (Kerdraon \& Delouis 1997).

Radio spectral data are obtained from different ground-based observatories and from the space. Radio dynamic spectra are recorded in European daytime at decimeter-meter wavelengths in Ondrejov, Czech Republic (Jiricka et al. 1993), and in Potsdam-Tremsdorf (AIP), Germany (Mann et al. 1992). The Potsdam instrument consists of four different aerials and produces spectrograms in the range from 40 to $800 \mathrm{MHz}$, with a sweep rate of $0.1 \mathrm{~s}$. The Astronomical Institute in Ondrejov provides the radio spectral data in the decimetric frequency range, from $800 \mathrm{MHz}$ to $2 \mathrm{GHz}$ and with a time resolution of $100 \mathrm{~ms}$, obtained from the radio spectrograph. It also contains a $3 \mathrm{GHz}$ radiometer with a time resolution of $10 \mathrm{~ms}$.

For American daytime, we use the microwave data obtained with the Owens Valley Solar Array (OVSA; Hurford \& Gary 1989). The OVSA data were sampled at 45 frequencies in the range of $1-18 \mathrm{GHz}$ every $12 \mathrm{~s}$. The Holloman dynamic spectra are recorded in New Mexico, USA (a part of the Radio Solar Telescope Network operated by the US Air Force). The
Holloman spectral data is from a swept frequency receiver operating in the frequency range from 25 to $180 \mathrm{MHz}$.

The low frequency, decametric data are taken from the WAVES receivers on the Wind spacecraft. The spectral data range in frequency is from 1.075 to $13.825 \mathrm{MHz}$ (Bougeret et al. 1995). This step-tuned superheterodyne receiver, which is connected to a dipole antenna in the spacecraft spin plane, sweeps 256 frequency channels in $16.128 \mathrm{~s}$ with a frequency resolution of $50 \mathrm{kHz}$ and a bandwidth of $20 \mathrm{kHz}$.

Hard X-ray light curves were observed with the Reuven Ramaty High Energy Solar Spectroscopic Imager (RHESSI; Lin et al. 2002). The hard X-ray light curves are derived from the Observing Summary Plot using the RHESSI software. Soft $\mathrm{X}$-ray imaging was done with the soft X-ray telescope (Tsuneta et al. 1991) on board the Yohkoh satellite (Ogawara et al. 1991). We also use the GOES X-ray data and the $\mathrm{H} \alpha$ flare data provided by NOAA.

The line-of-sight magnetograms from the Michelson Doppler Imager on SOHO (Scherrer et al. 1995) are used for the computations of coronal magnetic field with the potential-field source-surface (PFSS) model by Schatten et al. (1969). We use the PFSS Solarsoft package (Schrijver \& DeRosa 2003) available at http://www.lmsal.com/ derosa/pfsspack/.

\section{Overview of the Eruptions}

The GLEs under consideration, GLE 56 on 1998 May 2 and GLE 67 on 2003 November 2, were moderately strong, with a step-like onset delayed with respect to the impulsive phase of the corresponding flare. Figure 2 illustrates the associated active regions and structures. On 1998 May 2, the $\mathrm{H} \alpha$ flare was not far from the disk center, at S15W15 in NOAA AR8210 (region $\mathrm{A}$ in the middle left panel). This active region was interconnected to AR8214 (N25E25; region B) by a large-scale loop structure that was later observed to form part of the CME. Flaring was also present in the northern region, starting slightly earlier than in AR8210 (for more details, see Pohjolainen et al. 2001). The 2003 November 2 flare was closer to west limb, at S14W56 in AR10486. Coronal dimming areas, seen in the EIT difference images between regions $\mathrm{A}$ and $\mathrm{B}$, are "footprints" of departing CMEs (Thompson et al. 2000). Note a remarkable similarity between the eruptions' geometry in terms of relative location of flares indicated in the middle row, open magnetic flux tubes seen in the lower row, and CMEs mapped with EUV dimming areas in the upper row.

The two eruptions, even being qualitatively similar to each other, strongly differ in the total energetics. The 1998 May 2 event is associated with the X1.1/3B solar flare and moderately fast CME (the extrapolated second-order speed at the Sun is about $1240 \mathrm{~km} \mathrm{~s}^{-1}$ ). The 2003 November 2 event is associated with the $\mathrm{X} 8.3 / 2 \mathrm{~B}$ solar flare and very fast CME (the extrapolated speed $\approx 2660 \mathrm{~km} \mathrm{~s}^{-1}$ ).

Time profiles of the two soft X-ray flares, CMEs and GLEs are compared in Figure 3. The GLE appearance at a particular station depends on the asymptotic viewing direction and cut-off rigidity at the geographic location of the station. For the upper panel of Figure 3, we have selected for each event a polar station that reveals the earliest rise of the NM count rate. For a polar station, the cut-off rigidity is low, which makes the GLE signal stronger because of typically steep rigidity spectra, while the fast rise of GLE suggests that the viewing direction is not far from the particle flux axis. The latter implies that the observed GLE onset is the least affected by the particle 
2 May 1998

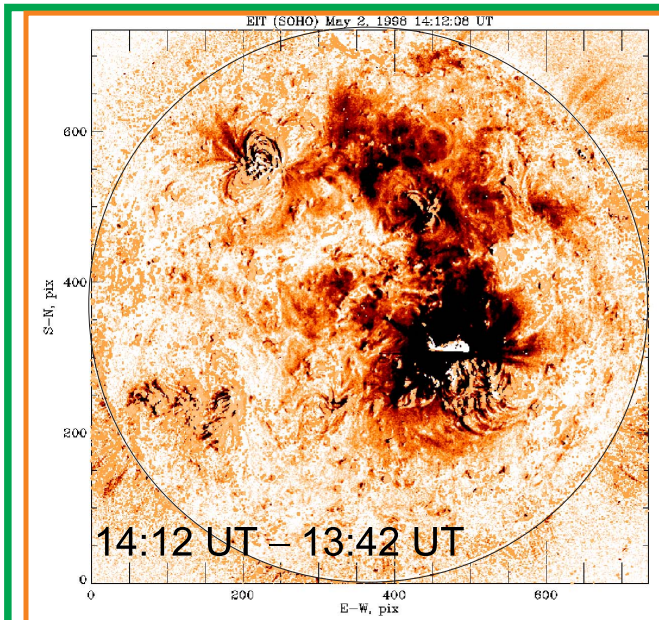

\section{Nov 2003}
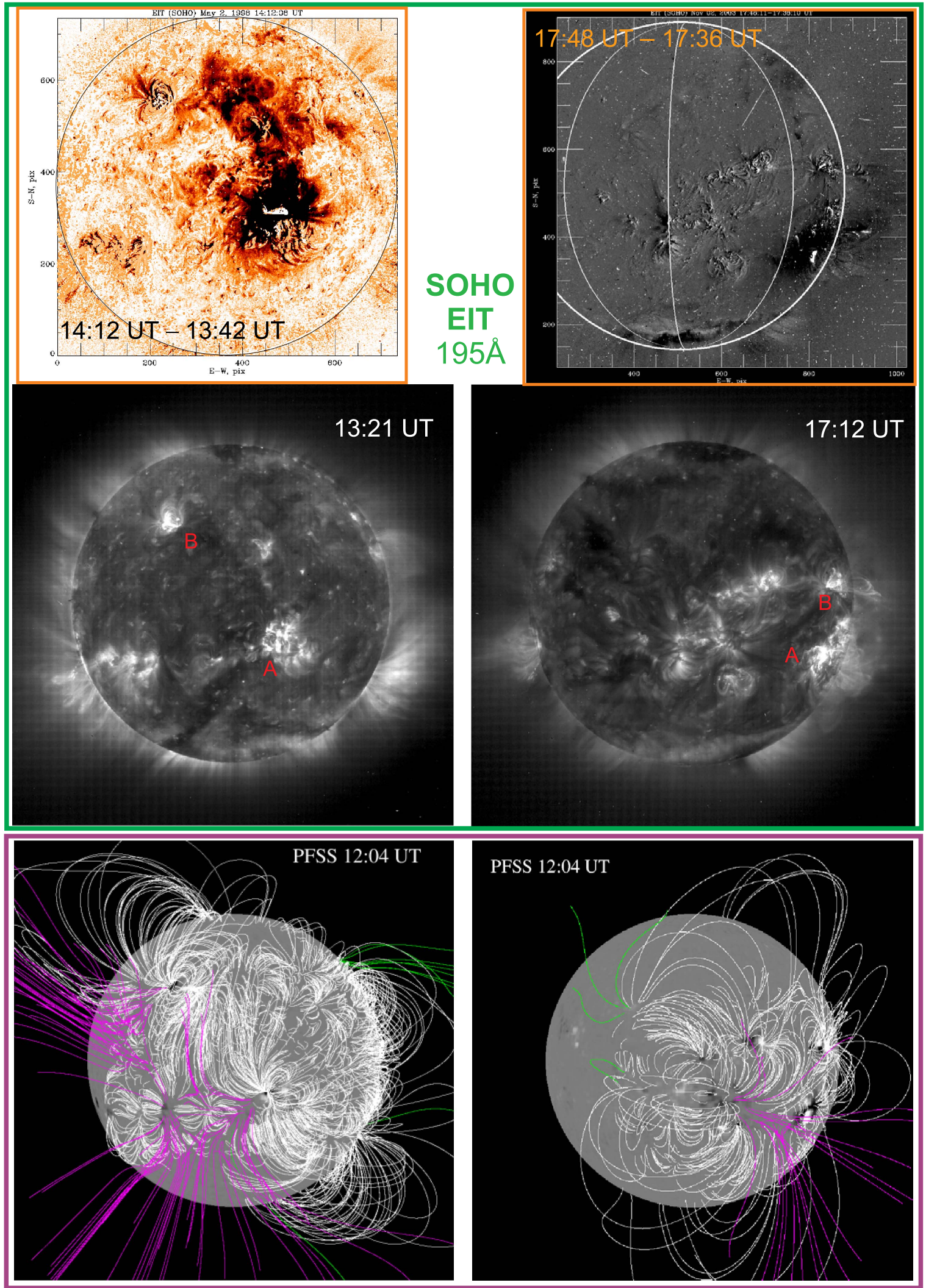

Figure 2. Global structure of the two eruptions. Lower row: coronal magnetic field before the eruption (computed with the PFSS model). Two upper rows show the $\mathrm{SOHO} /$ EIT images of the Sun: the EUV images before the eruption are in the middle row and the difference images revealing the post-eruption dimming areas are in the upper row. Flaring active regions are labeled with A. Coronal dimming area is seen between the active regions A and B. On 2003 November 2, the dimming is seen also above the west limb and near the south pole. Note: the upper right image is affected by solar energetic particles, which have arrived at 1 au by 17:48 UT. 

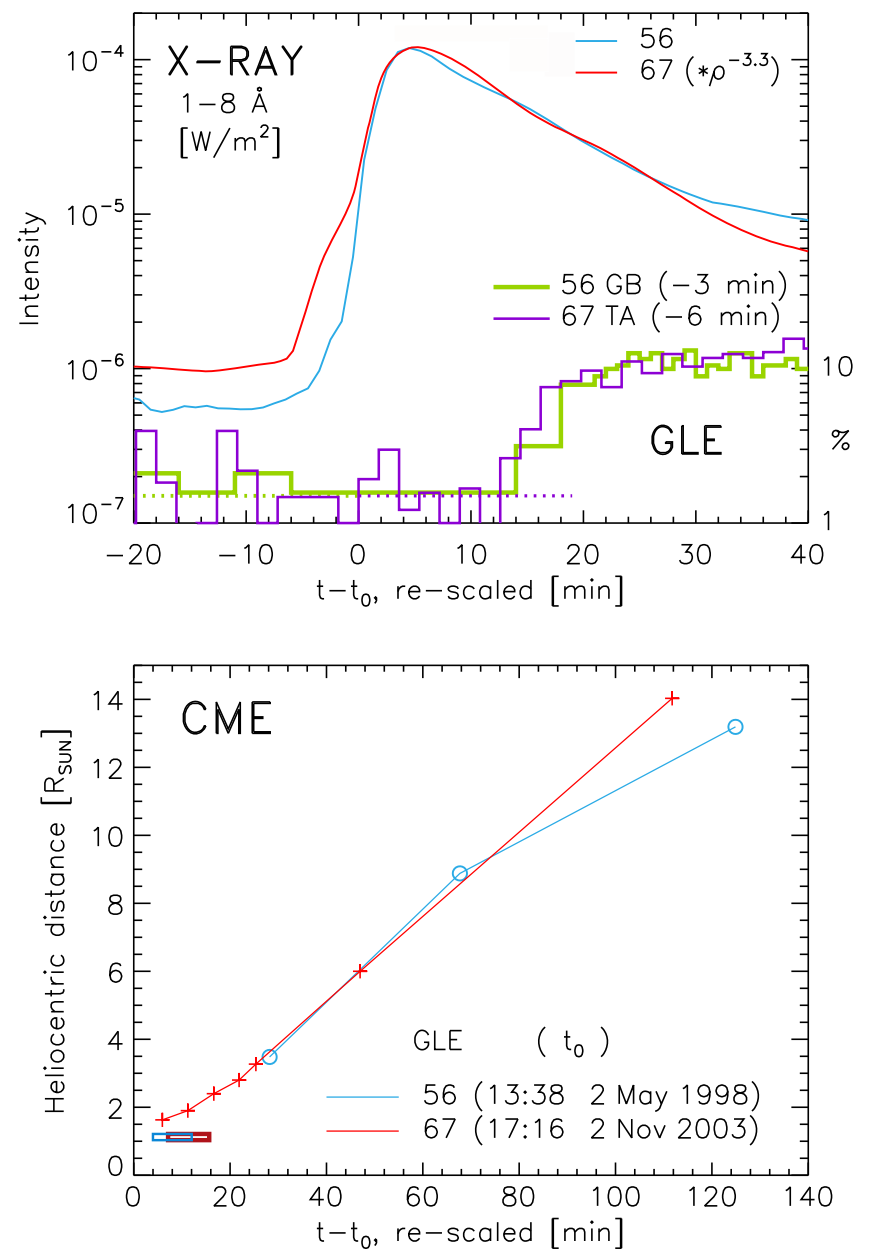

Figure 3. Time profiles of the NM count rate (GLE) and soft X-ray flare (upper panel) and the CME height (lower panel) for the events of 1998 May 2 and 2003 November 2 (GLEs 56 and 67). Timescale of the latter event is either stretched out with the scaling factor $\rho=1.8$, for CME and GLE, or compressed with the same scaling factor, for the soft X-ray flare. Abbreviations GB and TA in the upper panel stand for the Goose Bay and Terre Adelie stations, respectively. In both GLE profiles, $98.5 \%$ of the Galactic cosmic-ray (GCR) background is subtracted (the remnant background of $1.5 \%$ is shown with a dotted line). For a comparison with the 1 au observed electromagnetic emissions, timing of each GLE is corrected for a difference of the Sun-Earth transport time between protons and photons as indicated in brackets (the correction depends on the interplanetary transport conditions in a particular event; see Section 4 and Appendix C). For the 2003 November 2 CME, the part of the height-time profile below $3 R_{\odot}$ is from the MLSO Mk4 difference images, while the rest is from $\mathrm{SOHO} / \mathrm{LASCO}$, both at the position angle of $263^{\circ}$ (Section 5). Blue and red bars show the timing of the CME-launchassociated microwave bursts $C_{1}$ to be introduced in Section 5 . The reference time values, $t_{0}$, are given in the lower panel.

scattering in the interplanetary medium. The GLE timing in the upper panel is corrected for a delay due to the Sun-Earth transport time of protons arriving close to the flux axis.

In the considered event pair, the time delays between the flare impulsive phase (steep rise of the soft X-ray emission), on the one hand, and the CME arrival at a particular altitude and the GLE rise time at the Sun, on the other hand, are organized according to the reciprocal of an average speed of CME. For this reason, the corresponding time profiles of two eruptions nearly coincide if the time axis for one of the eruptions is appropriately rescaled. In the lower panel of Figure 3, the height-time profile of the 2003 November 2 CME is plotted versus the time, rescaled as $\left(t-t_{0}\right)_{\text {re-scaled }}=\left(t-t_{0}\right) \times \rho$, where the scaling factor (CME speed ratio) $\rho=1.8$, which makes the height-time profiles of the two CMEs nearly coincide up to $\approx 10 R_{\odot}$. The same scaling holds for the GLE rise profiles (upper panel).

We have also found that the soft X-ray flare profiles coincide if the timescale of the 2003 November 2 flare is changed in the opposite way, not stretched but compressed with the same scaling factor $\rho=1.8:\left(t-t_{0}\right)_{\text {re }- \text { scaled }}=\left(t-t_{0}\right) / \rho$, and the flare magnitude is rescaled as $\rho^{-3.3}$ (upper panel). In other words, in the considered pair of flare-CME events, the CME speed, $V_{\mathrm{CME}}$, is proportional to the soft $\mathrm{X}$-ray flare duration or to a power of the soft X-ray flare magnitude, $I_{\mathrm{X}}: V_{\mathrm{CME}} \sim I_{\mathrm{X}}^{0.3}$. After the submission of our paper, the latter scaling was independently reported for a statistical sample of eruptions associated with the $>10 \mathrm{MeV}$ proton events: $V_{\mathrm{CME}} \sim I_{\mathrm{X}}^{S}$ with $S=0.30 \pm 0.04$ (see Takahashi et al. 2016, and the discussion therein).

Importantly, neither re-scaling would result in the profile coincidence, if the reference time was taken not at the flare's impulsive phase, i.e., the flare time is an allotted instant of time for the entire eruption. In the two considered events, the flare pulse precedes CME, being a kind of triggering event, even though there was a development before that. Figures 2 and 3 mean for us that the two eruptions are morphologically homologous. Figure 3 also suggests that the relativistic proton emission (GLE) rises at the Sun when the CME leading edge (nose) travels from $2.2 R_{\odot}$ to $2.7 R_{\odot}$.

In the case of the 1998 May 2 GLE, the excess of the 5 -minute count rate of the Oulu neutron monitor was up to $6.5 \%$ over the galactic background. Surprisingly, there was no corresponding signal at the Apatity station, despite it being situated not very far from Oulu (e.g., Danilova et al. 1999). The difference at the two stations implies a high, beam-like anisotropy of the arriving solar particle flux. In the deka$\mathrm{MeV}$ range, the proton pitch angle distribution was accurately measured with the particle telescope ERNE/HED on SOHO (Torsti et al. 2004). The distribution was extremely narrow (Figure 4, right panel). To the best of our knowledge, it is the narrowest distribution ever observed.

For a comparison of the energetic particle production with the associated solar electromagnetic emissions, one has to infer from the 1 au observations the particle source profile at the Sun. In the general case, the source profile can be obtained by fitting the observed particle time-intensity profile and pitch angle distribution with a particle injection and interplanetary transport model. However, in such an anisotropic event as the 1998 May 2 is, the interplanetary transport modeling can be replaced with a simple shifting of the 1 au profile back to the Sun for the flight time appropriate to the particle energy. The time shifting method can reproduce well the rise and the maximum phase of the solar source in strongly anisotropic events but anyway shall not be used for the event's decay phase (e.g., Kocharov et al. 2015).

In Figure 4, we plot the time-shifted profiles of the NMobserved relativistic protons and the ERNE-observed $\sim 120 \mathrm{MeV}$ protons and $\sim 70 \mathrm{MeV}$ nucl $^{-1}$ helium. Each profile is shifted back to the Sun for the particle transport time but then eight minutes are added to the solar time for further comparison with electromagnetic emissions observed at $1 \mathrm{au}$. It is seen that all the particles are simultaneously emitted from the Sun, irrespective of particle species and energy. Despite an order of 

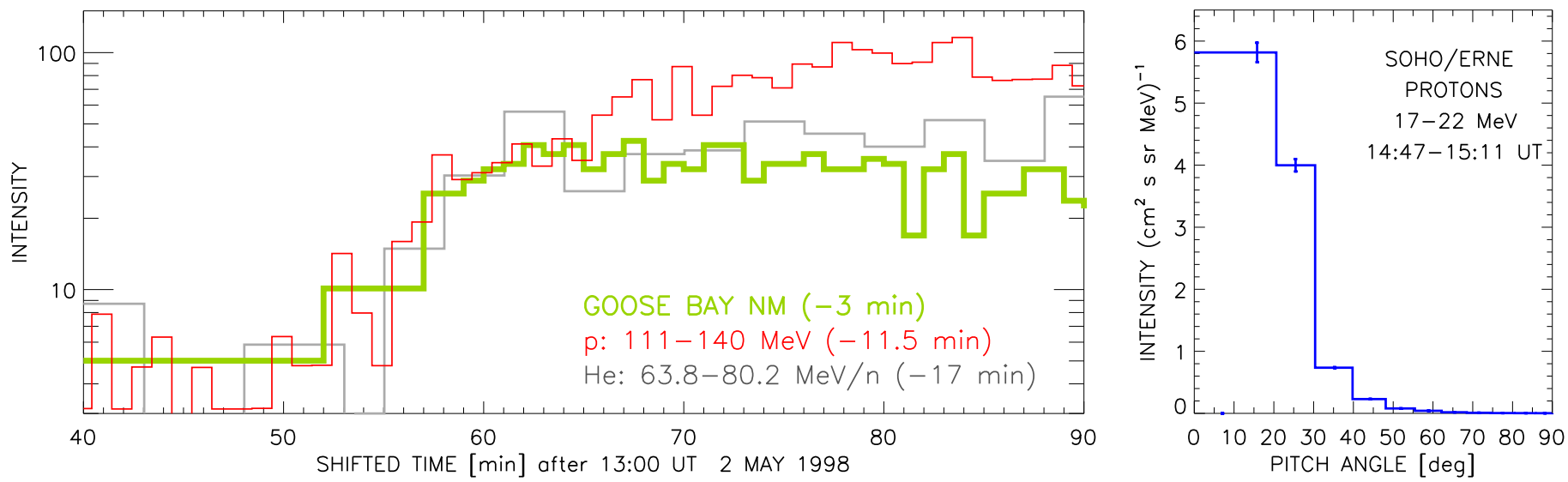

Figure 4. 1998 May 2 solar particle event: time-intensity profiles of high-energy protons and helium (left) and pitch angle distribution of deka-MeV protons (right). The time-intensity profiles are renormalized and shifted back for the time of the Sun-Earth transport along the spiral IMF line, but with 8 minutes added to the solar time. The count rate profile of the Goose Bay NM is shown with the GCR background reduced but not completely subtracted.

magnitude difference in the particle energy, no earlier appearance of lower energy particles is seen, in contrast to what would be expected at a gradual particle acceleration and concurrent escape to the solar wind. Note that this holds only for the high-energy particle component dominating above $50 \mathrm{MeVnucl}^{-1}$, while that component was not the only component observed in the event (Kocharov et al. 2007).

The 2003 November 2 event was characterized by a significant anisotropy in its initial phase. However, the pitch angle distribution of solar protons could not be a kind of narrow beam, because, for example, the Oulu NM count rate rose early in the event, despite the fact that the viewing direction of the station at that time was in the anti-Sun hemisphere (Figure 1). Obviously, the proton angular distribution of the 2003 November 2 GLE was not very narrow and, hence, the modeling of the particle transport from the solar source to 1 au shall be applied.

\section{Interplanetary Transport Modeling}

A standard model of the SEP transport in the IMF assumes that particles propagate along the guiding, large-scale magnetic field and experience a differential (small-angle) scattering due to the weak small-scale field, with any cross-field transport neglected. Such particle transport is described by the FokkerPlanck formalism (e.g., Earl 1981; Toptygin 1985), with the particle transport equation written as

$$
\frac{\partial f}{\partial t}+\mu V \frac{\partial f}{\partial \xi}=\frac{1}{2} \frac{\partial}{\partial \mu}\left(1-\mu^{2}\right) \nu \frac{\partial f}{\partial \mu}+\frac{V}{2 B} \frac{\partial B}{\partial \xi}\left(1-\mu^{2}\right) \frac{\partial f}{\partial \mu},
$$

where $f, V$, and $\mu$ are particle distribution function, speed, and pitch-angle cosine, $\mu=\cos \alpha$; the coordinate $\xi$ is the fieldaligned distance; the Fokker-Plank coefficient of pitch-angle scattering is

$$
\nu=\frac{3(V / \lambda)}{(2-q)(4-q)}|\mu|^{q-1}
$$

$q$ is spectral index of plasma turbulence that scatters the particles; and $\lambda$ is the particle mean-free path associated with that scattering. The magnetic field derivative is often expressed in terms of the focusing length, $L=-B /(d B / d \xi)$. The volumetric number density $f$ may be replaced with the linear number density $F=f / B$, the number of particles per unit of the magnetic flux tube length.

Provided that the ratio $\lambda / L$ does not change with distance, the exact solution of the steady-state Boltzmann equation in arbitrary guiding field configurations is (Kunstmann 1979; Earl 1981)

$$
F=A \exp \left[\frac{4-q}{3} \frac{\lambda}{L} \mu|\mu|^{1-q}\right] .
$$

If the ratio $\lambda / L$ still changes with distance from the Sun, Equation (3) provides only an approximate solution for the case of strong scattering, $\lambda \ll L$. An exact steady-state solution exists also in the opposite case of the weak scattering (Toptygin 1985, Section 11.1):

$$
f(\xi, \alpha)=\frac{B(\xi)}{\pi B\left(\xi_{0}\right) \sigma^{2}(\xi)} \exp \left(-\frac{\alpha^{2}}{\sigma^{2}(\xi)}\right),
$$

where the distribution width

$$
\sigma^{2}(\xi)=4 \int_{\xi_{0}}^{\xi} \frac{B(\xi) d s}{B(s) l(s)}
$$

and $l$ is the scattering length for particles moving in the parallel to magnetic field direction. A relation between $l$ and $\lambda$ depends on the angular dependence of the scattering frequency, the power-law index $q$ in Equation (2). At $q=1$, in particular, scattering is isotropic and $l=2 \lambda$.

In the radial magnetic field $\left(\xi=r, B \sim 1 / r^{2}\right)$, at isotropic scattering and constant (radial) mean-free path $\left(\lambda=\lambda_{r}\right)$, the distribution width far from the Sun $\left(r \gg R_{\odot}\right)$ in the weak scattering regime will be

$$
\sigma^{2}(r)=\frac{2}{3} \frac{r}{\lambda_{r}}
$$

while in the strong scattering regime, the pitch angle distribution is of the form

$$
F(\alpha)=A \exp \left(\frac{2 \lambda_{r}}{r} \cos \alpha\right) .
$$


An interpolating estimate for a moderate scattering could be

$$
\begin{aligned}
& F(\alpha)=A^{\prime} \exp \left(2 \frac{\cos \alpha-1}{\sigma^{2}}\right), \\
& \sigma^{2}(r)=\frac{r}{\lambda_{r}}\left[1-\frac{1}{3} \exp \left(-\frac{r}{\lambda_{r}}\right)\right],
\end{aligned}
$$

which can be used at any value of the particle mean-free path.

Mean-free path value in a particular event can be estimated from the proton flux anisotropy data. For instance, for the first period of the 2003 November 2 event, 17:35-17:45 UT, we estimate the width of the proton distribution to be $\sigma^{2} \approx 1.5$ $\operatorname{rad}^{2}$ (Appendix B), which would correspond in the radial transport model to the mean-free path $\lambda_{r}=0.54$ au. The meanfree path value determines the average value of the particle pitch-angle cosine over the Sun-Earth trip, and hence, the particle transport time from the Sun. Given the mean-free path, the particle transport equation can be solved, e.g., with Monte Carlo modeling, and the interplanetary transport functions and the solar source profile are found.

It is known that very different interplanetary transport models may be described by one and the same transport equation and thus result in one and the same intensity-time profile of particles at $1 \mathrm{au}$. For instance, the focused 1D diffusion along a spiral magnetic field line of the Parker's model of solar wind can be reformulated as a radial diffusion with a renormalized diffusion coefficient (e.g., Kunow et al. 1991; Kocharov et al. 1998). The same equation of the radial diffusion describes also the focused $1 \mathrm{D}$ diffusion in the radial magnetic field and the spherical 3D diffusion with isotropic diffusion tensor. However, at one and the same transport equation (and, hence, the same transport time), the symmetry axis of the particle angular distribution may be different. At the isotropic diffusion tensor, the particle distribution axis would be parallel to the Sun-Earth direction, while in the case of focused 1D diffusion in the Parker's solar wind, the axis is parallel to the field line.

Direction of the distribution axis may be altered by the particle transport across magnetic field lines that is ignored in the 1D transport model described by Equation (1). Cross-field transport can be caused by particles following random-walking field lines, decoupling from the field lines, and drifts due to large-scale gradients. In the framework of the diffusionconvection equation of cosmic-ray transport (Parker 1965; Toptygin 1985), these are typically modeled through a diffusion tensor that has diffusive components across the mean magnetic field, and asymmetric elements describing the drifts. The cross-field diffusion coefficients have recently been implemented to the Fokker-Planck formalism (e.g., Zhang et al. 2009; Dröge et al. 2010) and the cross-field diffusion has been shown to extend the SEP events in longitude and latitude considerably. Drifts, while usually ignored for SEPs, have recently been shown to have a significant effect on both the cross-field distribution and energies of the SEPs, in particular, at GLE energies at late stages of the event (Dalla et al. 2013). However, it is not presently expected that a realistic cross-field transport can make the SEP diffusion tensor completely isotropic as the 3D spherical diffusion model assumes.

Some solar particle events were observed inside magnetic clouds of previous CMEs, and the particle transport was correspondingly modeled in a kind of interplanetary magnetic loop (Kocharov et al. 2005; Ruffolo et al. 2006). However, for the 2003 November 2 event, an interplanetary magnetic cloud has not been identified with in situ plasma data, even though the solar wind was strongly distorted by previous solar eruptions (Appendix A). Perhaps because of the distorted solar wind conditions, the 2003 November 2 GLE itself is rather difficult for the analysis. The fitting of the NM network data of that event is described in detail in Appendix B.

With a still limited number of stations and because of the presence of the isotropic component in the 2003 November 2 GLE, a determination of the particle flux axis in that event is ambiguous. It is possible that the flux axis points $\approx 15^{\circ}$ off the radial, anti-sunward direction (the case shown in Figure 1), which corresponds to the best fit that satisfies all additional conditions listed in Appendix B. However, if we allow up to a $10 \%$ increase in the sum of differences between the modeled NM responses and the observed NM responses, $\mathcal{D}$ defined by Equation (13), the available data can be explained with the particle flux axis pointing anywhere along a strip extending for $\approx 70^{\circ}$, from the direction almost from the Sun to a direction almost from the Earth's south pole, at one and the same width of the pitch angle distribution. In particular, it is possible that the flux axis for a five minute bin is only $\approx 15^{\circ}$ off the IMF direction measured on $A C E$, which does not seem to be a large deviation, especially for the observed variance of the IMF direction itself (Appendix A).

The uncertainty in the flux direction implies an uncertainty in the interplanetary transport model applicable to the event. For this reason, we have considered two alternative, extreme models of the interplanetary transport: (1) a focused 1D transport along the standard, spiral IMF line of the $500 \mathrm{~km} \mathrm{~s}^{-1}$ solar wind and (2) a radial transport that is similar to the 3D radial diffusion (Appendix C). It turns out, however, that the inferred solar source profile only weakly depends on the adopted transport model, if the width of the pitch angle distribution at $1 \mathrm{au}$ is fixed.

In the left panel of Figure 5, we show the solar source of relativistic protons as inferred with the standard solar wind model. Also shown is the corresponding, simulated intensitytime profile of relativistic protons arriving at $1 \mathrm{au}$. The source profile and the parallel mean-free path $\lambda$ are adjusted to fit (1) the count rate profiles observed by the entire network of neutron monitors in 5-minute bins and (2) the 1-minute data of the McMurdo neutron monitor as shown in this figure. The model pitch angle distribution is in the right panel. It comprises the particles streaming from the Sun and the isotropic component. The simulation is performed in the frame of a modified model of focused transport. The modifications are explained in detail in Appendix C. They account, in particular, for the observed feature of the 2003 November 2 GLE that is the isotropic component rising very early in the event. Such a feature can be reproduced with a large-angle (integral) scattering process added to the standard model. More modeling results are shown in Appendix $\mathrm{C}$ and the next section.

\section{Associated Electromagnetic Emissions}

An overview of radio emissions associated with the 1998 May 2 eruption is shown in Figure 6. The impulsive phase of the flare comprises two episodes, $F_{1}$ and $F_{2}$. They are associated in the $432 \mathrm{MHz}$ images with two small, loop-like sources connected to AR8210 (the NRH observation, not shown in our figures). The flare bursts $F_{1}$ and $F_{2}$ are followed by the burst $\mathrm{C}_{1}$ at $\leqslant 1.5 \mathrm{GHz}$. At $432 \mathrm{MHz}$, the strong and 

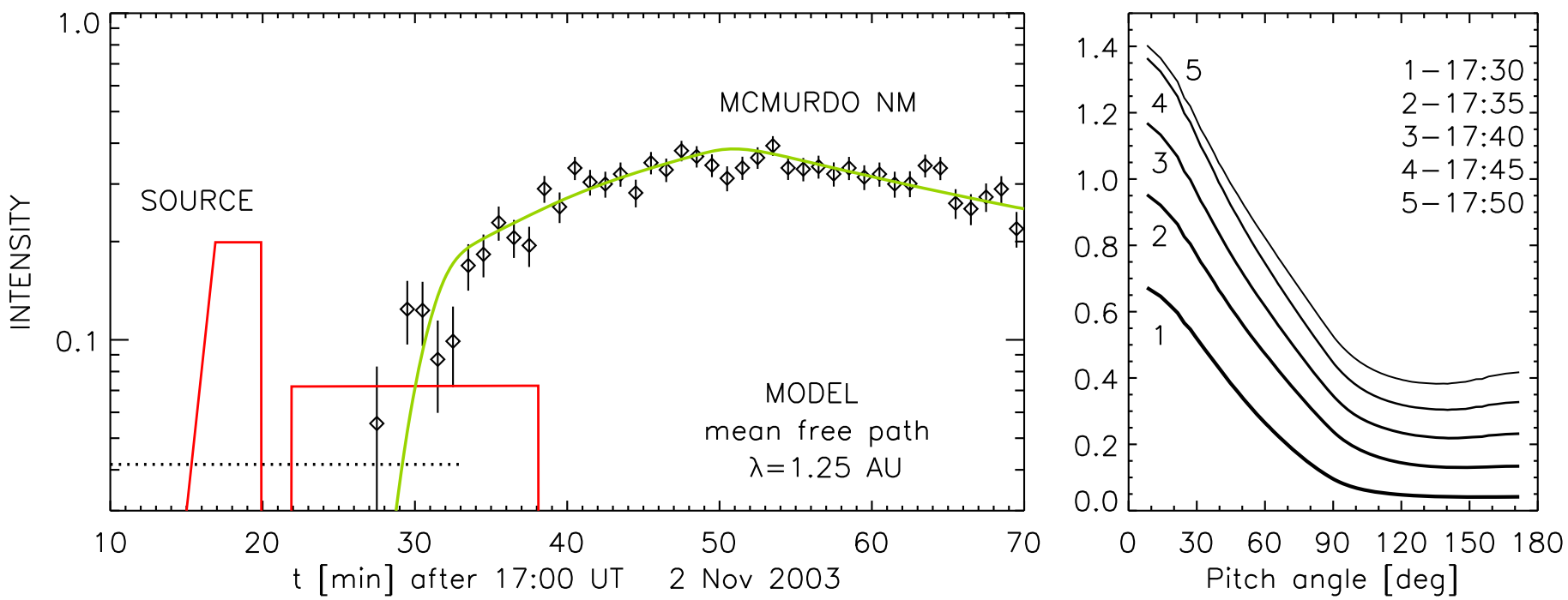

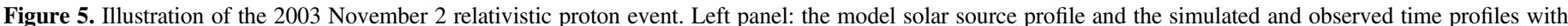

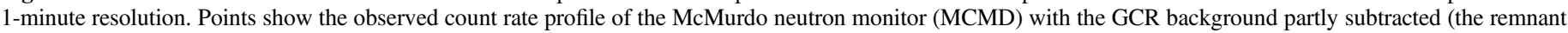

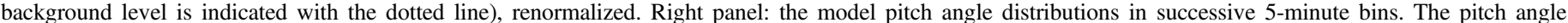

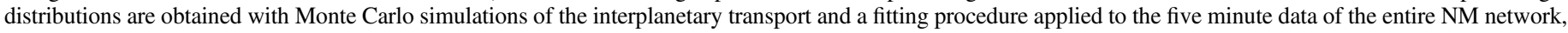

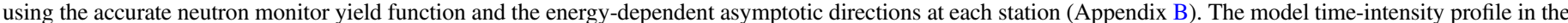

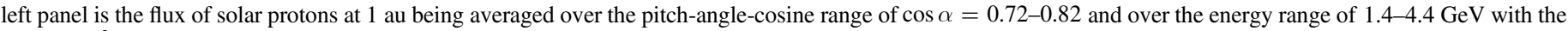
weight $E^{-3}$, plotted for a verification of the source time profile with 1-minute resolution.

compact source $\mathrm{C}_{1}$ is seen to the north from the previous, flare sources $F_{1}$ and $F_{2}$. In contrast to the flare bursts, burst $C_{1}$ does not have high-frequency counterparts. The $\mathrm{C}_{1}$ source appears first at 13:41:30 UT, around $450 \mathrm{MHz}$ in the Potsdam spectrum (also the PHOENIX-2 observation, Messmer et al. 1999), which is 1-2 minutes after the passage of the Moreton $(\mathrm{H} \alpha)$ wave at that (projected) location (Pohjolainen et al. 2001, Figure 6 therein). The global wave was also observed in EUV by $\mathrm{SOHO} / \mathrm{EIT}$, while with a lower cadence. The $\mathrm{C}_{1}$ source could be locally triggered by the global coronal wave but does not move with the wave.

The decimetric continuum $\mathrm{C}_{1}$ is followed by and partly coincides with the extended coronal source $\mathrm{C}_{2}$ seen in the NRH images at 236 and $164 \mathrm{MHz}$ (Figure 6). Pohjolainen et al. (2001) pointed out that the $\mathrm{C}_{2}$-source shape is close to the shape of coronal dimming later observed by EIT (upper left panel of our Figure 2). The $\mathrm{C}_{2}-\mathrm{CME}$ relation is additionally illustrated with Figure 7 . The radio source $\mathrm{C}_{2}$ could be produced by the rising $\mathrm{CME}$ during the $\mathrm{CME}$ motion nearly perpendicular to the magnetic field lines in the top sections of high coronal loops. Temporal and spacial relations between the sources $\mathrm{C}_{1}$ and $\mathrm{C}_{2}$, along with the lack of the $\mathrm{C}_{1}$ emission above $1.5 \mathrm{GHz}$ (in contrast to the flare bursts $F_{1}$ and $F_{2}$ ) allow us to propose that the source $\mathrm{C}_{1}$ should also be ascribed to CME.

The LASCO CME in this event is not very fast, the near-Sun speed is about $1200 \mathrm{~km} \mathrm{~s}^{-1}$. A weak, intermittent low-frequency type II burst is observed on Wind (Figure 6). We use the LASCO CME height-time, second-order profile to make a frequency-time curve for the observed fragments of type II. Two curves, for the fundamental and harmonic emissions, are calculated with eight times the model density of corona by Saito et al. (1977). The curves successfully connect the observed slow-drifting bursts and provide an extrapolation back to the Sun. Note that the applied fitting procedure is aimed only at formal interpolating and extrapolating of the observed bursts. For such a goal, we use a simple approximation that fits the radio data, while many other formal approximations could also do the job (for a pattern of non-formal, in-depth consideration, see, e.g., Krupar et al. 2016).

The extrapolated frequency-time track in Figure 6 meets no metric type II emissions visible in the Potsdam data. However, Pohjolainen et al. (2001) have analyzed the NRH data and revealed not far from that track a type-II-like burst at 13:42:30 UT-13:45:30 UT in the frequency range from 250 to $150 \mathrm{MHz}$. That burst was produced by a compact source that initially appeared to the north from $\mathrm{C}_{1}$ and then moved in the direction of the later observed white-light CME (burst M3 in their Figures 8 and 13). Based on the timing of the bursts $C_{1}$ and M3, we consider 13:42 UT as the earliest time of the CME launch in the 1998 May 2 event.

Lowermost panel of Figure 6 shows a couple of the GLE profiles with fastest and steepest onset, being shifted in time back to the Sun and with 8 minutes added for a comparison with observed electromagnetic emissions. The rise phase of the particle emission coincides with the disappearance of the global coronal, $\sim 0.8 R_{\odot}$, radio source $\mathrm{C}_{2}$, also coincides with the latest low-frequency type III bursts and the first episode of the decametric type II emission. The lower panel of Figure 7 shows that at the GLE onset time, 13:52 UT, the CME leading edge could be at the top of the helmet streamer in the southwest, over the flaring active region. All of these observations taken together mean for us that the first relativistic protons were injected into the interplanetary space during the CME exit from the high coronal structures, like large loops and helmet streamers, at episodes of magnetic reconnection with open magnetic field lines traced by the type III electrons.

In contrast to the 1998 May 2 event, the 2003 November 2 eruption is well observed in hard X-rays (RHESSI), and what is most important is that we have the high-cadence white-light images of the low corona at the time of the GLE onset (MLSO). Broadband radio emissions, hard X-ray and high-energy particle profiles of the 2003 November 2 eruption are summarized in Figures 8(a) and (b). 


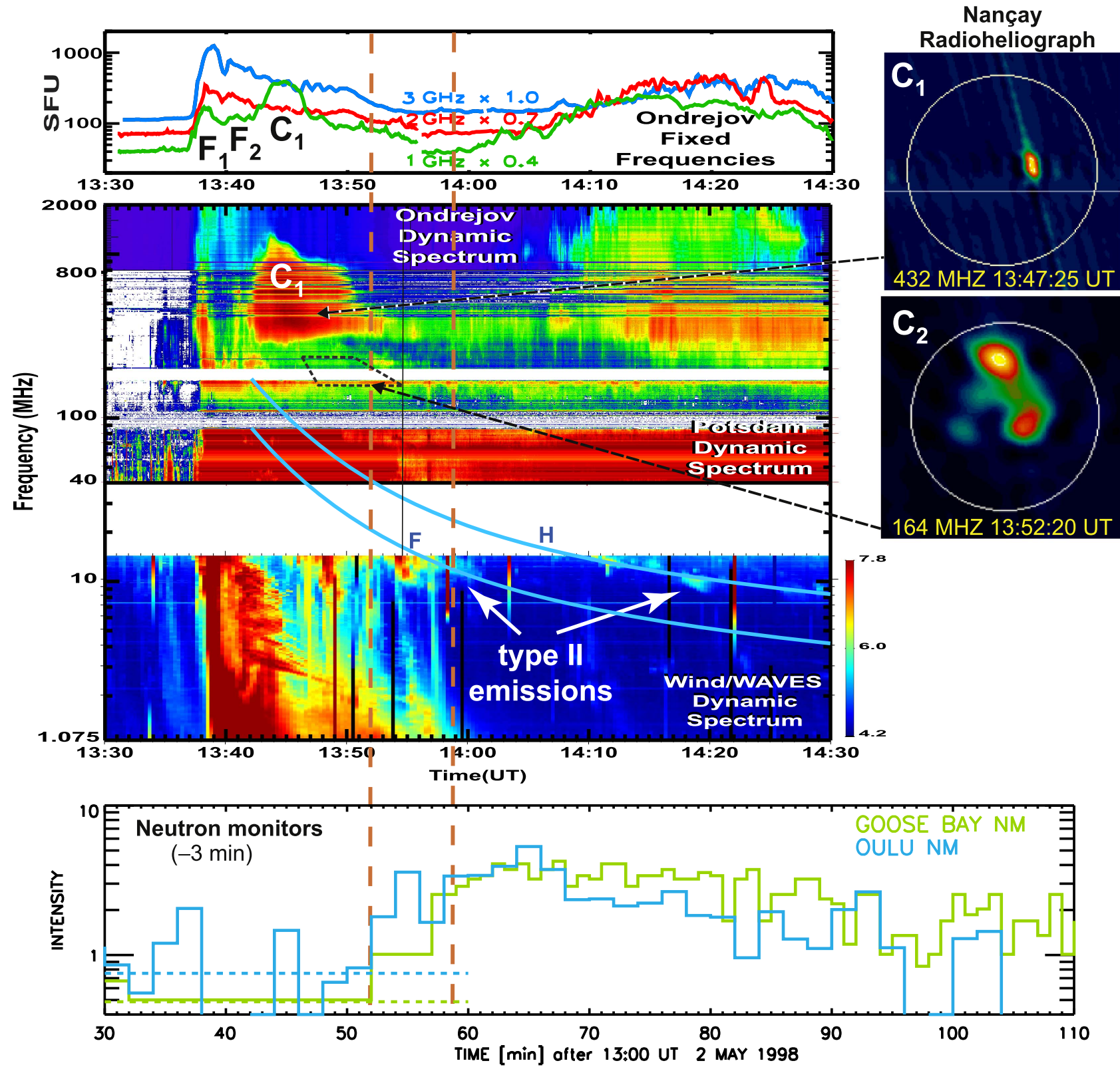

Figure 6. Radio emissions of the 1998 May 2 event (including the NRH images of sources $\mathrm{C}_{1}$ and $\mathrm{C}_{2}$ ) vs. the GLE profiles at two stations with the earliest and steepest onset (shown in the lowermost panel). Parallel sides of the dashed trapezoid in the middle panel indicate the time intervals when the radio source $\mathrm{C}_{2}$ is seen in the $\mathrm{NRH}$ images at 164 and $232 \mathrm{MHz}$. The GLE profiles are shifted by -3 minutes for a comparison with radio emissions. The rise phase of the relativistic proton emission coincides with the occurrence of the low-frequency type III bursts at 13:52-13:58 UT (Wind/WAVES). Two blue curves in the middle panel show the CME frequency-time track (the fundamental and harmonic emissions) that fits the type II fragments. Type II features were also observed later, during 14:30-14:50 UT and after 16:55 UT.

In the three upper panels of Figure 8(a), we compare the time profiles of the hard X-ray and microwave emissions. A doublepulse structure of the flare impulsive phase, $F_{1}-F_{2}$, is evident from the hard X-ray profiles, while microwave profiles at around $10 \mathrm{GHz}$ reveal equally strong pulses also during the next 5 minutes. The hard $\mathrm{X}$-ray to microwave emission ratio for selected peaks is also given in Figure 8(a) (numbers between the two upper panels). In terms of that ratio, the flare bursts are rich in the hard X-rays, while the post-impulsivephase bursts like $C_{1}$ are rich in microwaves and relatively poor in hard X-rays. The microwave richness of the latter bursts may imply energetic electrons trapped in high coronal structures (Lee et al. 2000).
We find that the microwave burst $\mathrm{C}_{1}$ coincides with the main period of the CME acceleration observed at MLSO (period $\mathrm{A}_{1}$ in the middle panel of Figure 8(a), also Figure 9). With a threepoint quadratic approximation of the $\mathrm{A}_{1}$ segment of the heighttime profile, we estimate a percentage of the maximum speed squared that had been gained by the CME by the beginning, middle, and end of the period $\mathrm{A}_{1},\left(V / 2660 \mathrm{~km} \mathrm{~s}^{-1}\right)^{2}$, shown at the CME height-time profile in Figure 8(a). It turns out that in terms of the gained kinetic energy, the period $\mathrm{A}_{1}$ is indeed the main period of the CME acceleration. Because the burst $\mathrm{C}_{1}$ coincides with the main period of CME acceleration and is relatively weak in hard X-rays, we identify the $\mathrm{C}_{1}$ burst as an event associated with the CME launch. 


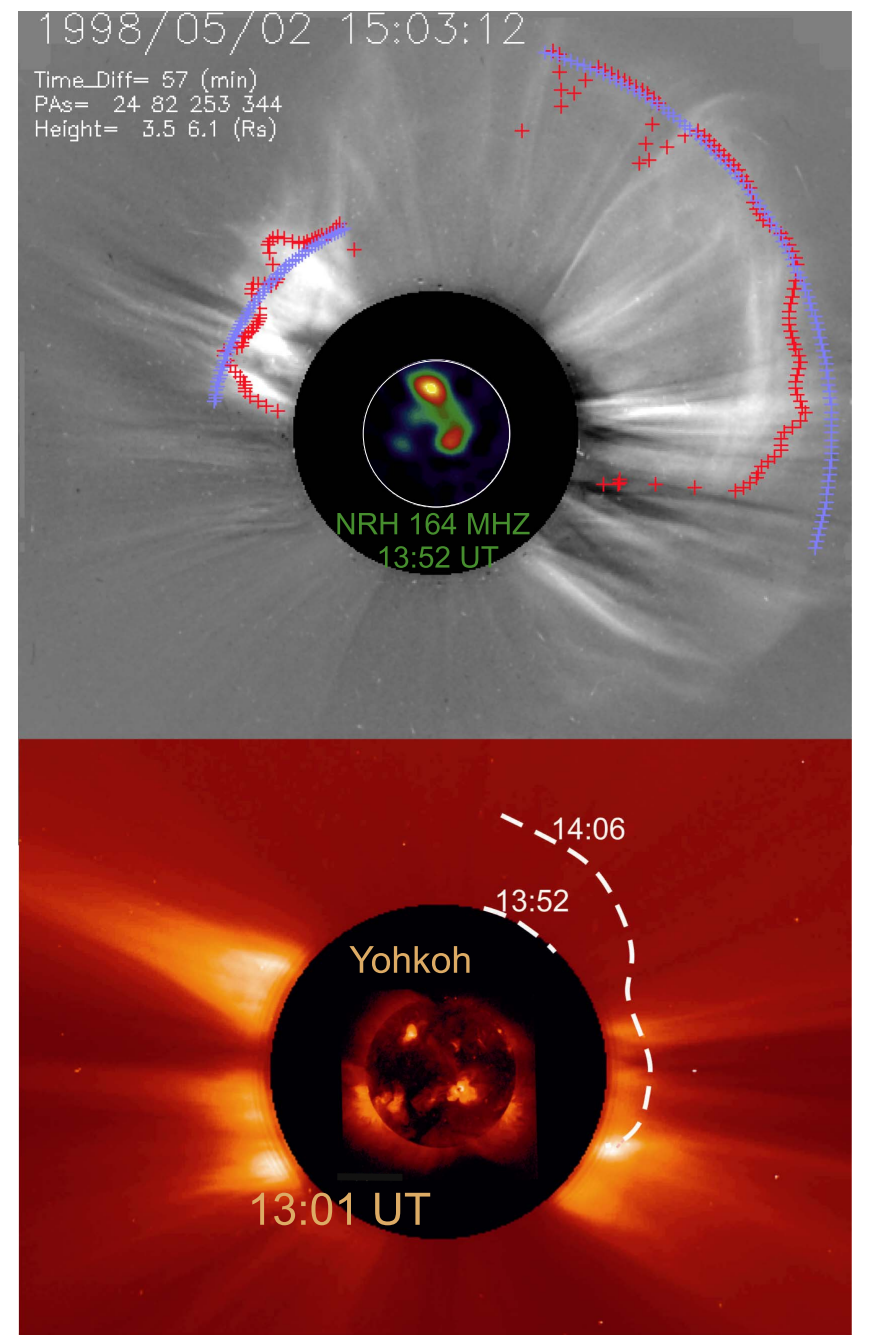

Figure 7. 1998 May 2 CME. Upper panel illustrates the CME geometry with the LASCO C2 difference image from GMU SEEDS and the radio map at $164 \mathrm{MHz}$ (source $\mathrm{C}_{2}$ ) from NRH. Lower panel: soft X-ray and white-light, coronagraph images taken shortly before the eruption. Dashed curves additionally show the CME leading edge outlined from the LASCO C2 running difference image at 14:06 UT and a segment of the leading edge extrapolated back to the GLE onset time, 13:52 UT.

In Figure 8(b), we compare the history of the relativistic proton release at the Sun with the dynamic radio spectra in the metric-to-hectometric frequency range. The proton source profiles (bottom panel) are inferred from the data of the neutron monitor network with modeling of the particle transport from the Sun to the detectors on the ground (Appendices B and C). The three versions of the particle source profile correspond to the three possible values of the proton mean-free path for the modeling of the interplanetary transport. They illustrate the actual uncertainty range. A source time profile was optimized to fit the first 25-30 minutes of GLE (red bar), even though it may also fit the GLE's second period (blue bar), as is evident from Figure 5. The proton energy spectrum in the first period of the event steepens with energy. The steepening, however, weakens with time and eventually disappears, so that the spectrum becomes a power law by the beginning of the second period (Appendix B).

A low-frequency type II radio burst is well seen in the Wind/ WAVES data after 17:30 UT. The plotted frequency-time track (curve pair in Figure 8(b)) is for the LASCO CME kinematics and the Saito et al. (1977) coronal density model. The curves approximate well the observed low-frequency type II burst and provide some extrapolation into the metric band. However, we cannot find clear evidence of corresponding type II emission in the Holloman spectrum.

The first pulse of the relativistic proton emission at 17:25-17:28 UT ( \pm 1 minute) coincides with the late type III bursts, which do not have high-frequency counterparts at frequencies $\gtrsim 150 \mathrm{MHz}$. The second part of the proton source, at $\sim 17: 30-17: 45 \mathrm{UT}$, coincides with a period of intensified emission of type II and the latest low-frequency type III bursts with no counterparts at $\gtrsim 25 \mathrm{MHz}$. A low and decreasing with time starting frequency of the CME-associated type III bursts differentiates them from the flare type III bursts observed during 17:15 UT-17:22 UT.

The eruption development is further illustrated with images of the solar disk and corona in Figures 10 and 11. The EIT difference images shown in the left and middle panels of Figure 10 reveal the disappearance of the high loop between 17:12 and 17:24 UT, shortly before the proton emission start. As is evident from the MLSO low corona images, the proton emission onset at 17:25 UT coincides with the CME arrival at tips of helmet streamers (Figures 8(b) and 9). After that, the CME expands both radially, into the solar wind and laterally, toward the south pole. Dotted line in the upper panel of Figure 11 is the CME-edge outline at 17:28 UT, plotted on the CME image at 17:25 UT. The lateral expansion starts at 17:25 UT and ends prior to 17:36 UT when the south pole dimming is observed (right panel of Figure 10). In the LASCO C2 frame at 17:54 UT (Figure 11), the CME's south flank is already over the south pole and the flank's brightness is enhanced. The lateral expansion of CME coincides with both the series of lowfrequency type III radio bursts and the first pulse of the relativistic proton emission.

Comparison of the MLSO and LASCO images in the upper panel of Figure 11 reveals a similarity between them in the northwest sector and two remarkable differences in the southwest sector, one of which is the already mentioned south-flank brightening. The second difference is the jet-like brightening in southwest. It appears in the sector of the previous slow, narrow injections shown in two lower panels of Figure 11. The new, jet-like brightening starts after 17:30 UT but before 17:54 UT. This period includes the intensified radio emission of type II, the latest type III bursts and the less intensive part of the proton source shown in the lowermost panel of Figure 8(b).

\section{Discussion}

\subsection{Comparison of Corpuscular and Radio Emissions}

In a correlative study between $>20 \mathrm{MeV}$ solar proton events, CMEs, and radio bursts, Cane et al. (2002) found that essentially all of the proton events are preceded by groups of type III bursts and all are preceded by CMEs. The authors identified the associated radio bursts as type III- $l$, which are long-lasting, intense bursts seen in the low-frequency observations made from space. In such events, open field lines extend from within $0.5 R_{\odot}$ into the interplanetary medium and the burst-generating electrons originate from the reconnection regions below fast CMEs. More recently, Gopalswamy et al. (2012) compared the onset time of low-frequency type III bursts and the onset time of GLEs, found out that they typically 

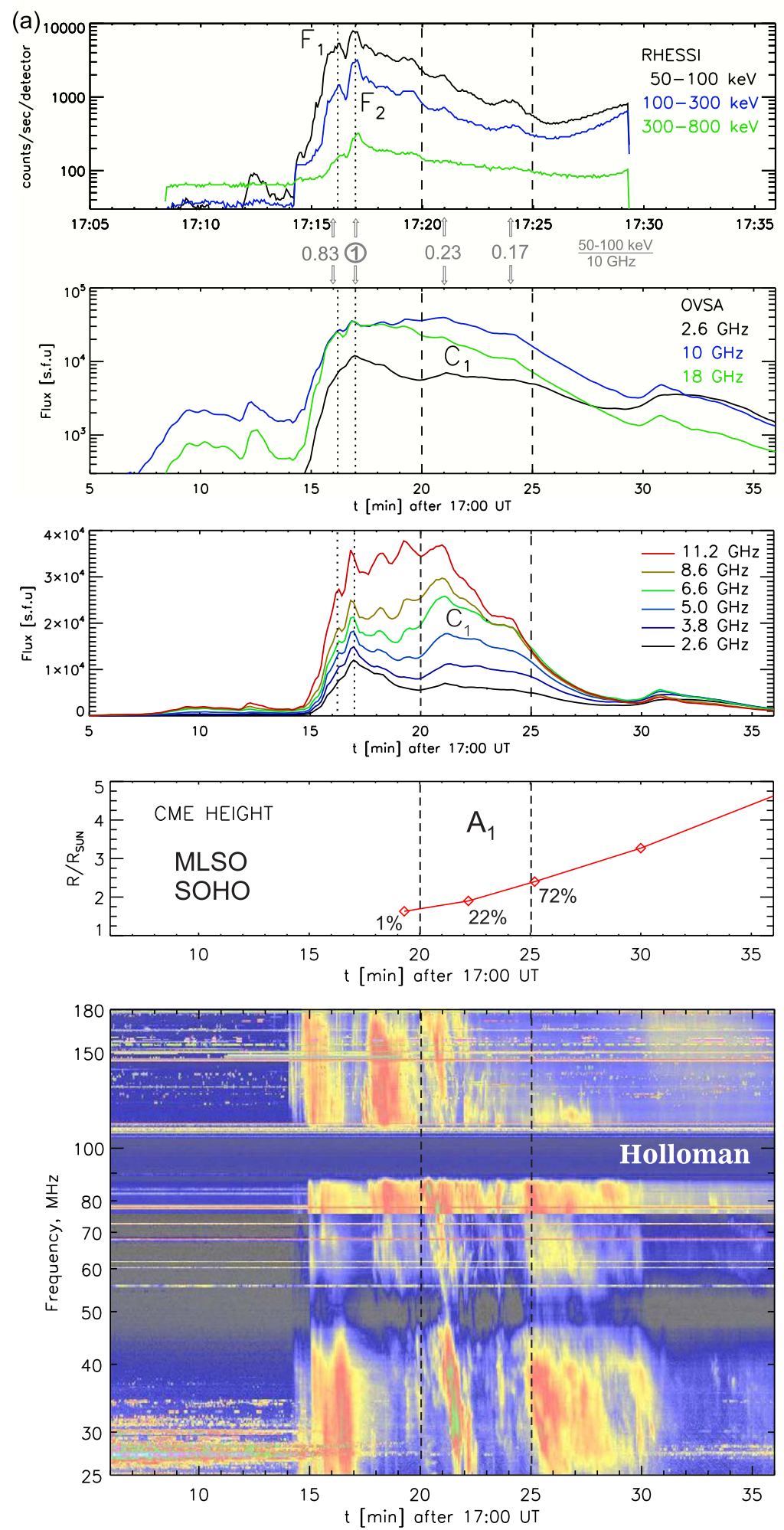

Figure 8. (a) The 2003 November 2 eruption at high frequencies: hard X-ray and microwave emissions (three upper panels) and dynamic radio spectrum in the metric band (lower panel). The numbers between the two upper panels indicate the X-ray to microwave emission ratio, normalized to the ratio at peak $\mathrm{F}_{2}$. Middle panel: the CME height-time profile (three first points are from the images shown in Figure 9, while the rest is from LASCO $/ \mathrm{C} 2$, both at the position angle of $263^{\circ}$ ). The numbers at the curve show a percentage of the CME-speed-squared gained by the beginning, center, and end of the period $\mathrm{A}_{1}$. The main acceleration period of CME, $\mathrm{A}_{1}$, coincides with the microwave burst $\mathrm{C}_{1}$. (b) The 2003 November 2 eruption at low frequencies (upper left and middle panels). Lower panel: the GLE illustrated with (1) the inferred proton source profile at the Sun (shifted for +8 minutes; shown for three possible values of the proton mean-free path, $\lambda$, for the model of focused transport in the Parker's solar wind) and (2) the observed count rate profile of the Terre Adelie neutron monitor (shifted back to Sun for the proton transport time and with 8 minutes added). It is seen that emission of relativistic protons starts upon the CME exit from the helmet streamers (upper right panel) and coincides with the low-frequency type III bursts. The late, less intensive part of the proton source also coincides with the initial, intensified part of the decametric type II emission seen at 17:30 UT-17:55 UT, while the type II burst continues until 01:00 UT of the next day at frequencies down to $250 \mathrm{kHz}$. 


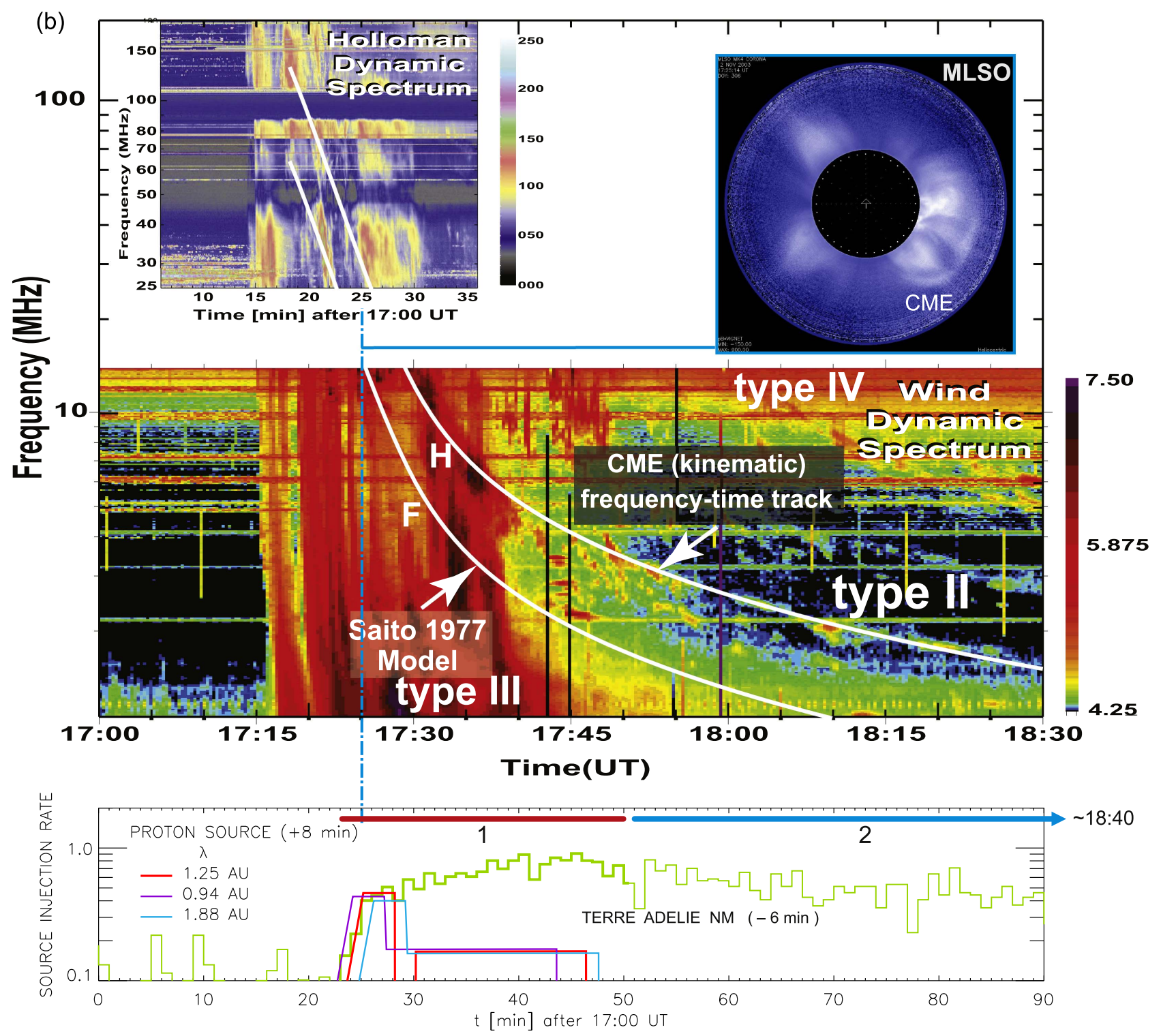

Figure 8. (Continued.)

do not coincide, and concluded that the high-energy protons and the type III electrons could not originate from one and the same source. Instead, we deduce and analyze the entire time profile of the SEP source.

Perviously, a similar approach was used for SEPs of lower energies. In a case study of the 2000 April 4 (non-GLE) event, Kocharov et al. (2010) deduced the source profiles of the $0.25-3 \mathrm{MeV}$ electrons and the $\sim 20 \mathrm{MeV}$ nucl $^{-1}$ helium. The inferred sources of electrons and helium each comprised of two successive sub-sources. The second sub-source was the main source of helium, with a high helium abundance, $\mathrm{He} / \mathrm{p} \sim 0.1$, that is typical for SEPs originating from impulsive flares (e.g., Cliver 1996). It emitted helium simultaneously with the late, low-frequency bursts of type III, 20-40 minutes after the flare impulsive phase. Those type III bursts had low starting frequencies and were also associated with the CME-driven decametric type II burst. A straightforward interpretation was that SEPs were initially accelerated in the eruption's heliumrich core (such as solar flare), trapped and later released at episodes of magnetic reconnection in the course of the upward motion and expansion of CME. In the same event, also observed was a helium-poor SEP component, $\mathrm{He} / \mathrm{p} \sim 0.01$, which was attributed to the CME itself.

In the present work, we have deduced the source profile of the $>400 \mathrm{MeV}$ proton emission responsible for a GLE event. The deduced source profile of relativistic protons in the 2003 November 2 event begins with a $\approx 5$ minute long pulse that coincides with the late, low-frequency type III radio bursts (Figure 8(b)). A similar time-coincidence is observed in the 1998 May 2 event (Figure 6). Those type III bursts occur at the time when CME reaches the top sections of highest loops and also expands in the lateral direction (Figures 8(b) and 11). Hence, the magnetic reconnection with open field that is responsible for the type III emission could occur at the CME flank. We also note a diminution in the multiple type III bursts in the frequency range from 5 to $10 \mathrm{MHz}$ (Figure $8(\mathrm{~b}$ ), 17:24-17:30 UT). A similar diminution was previously observed in other events and an interpretation was the type III electron propagation through the located-above structures of the CME (Reiner et al. 2008). 

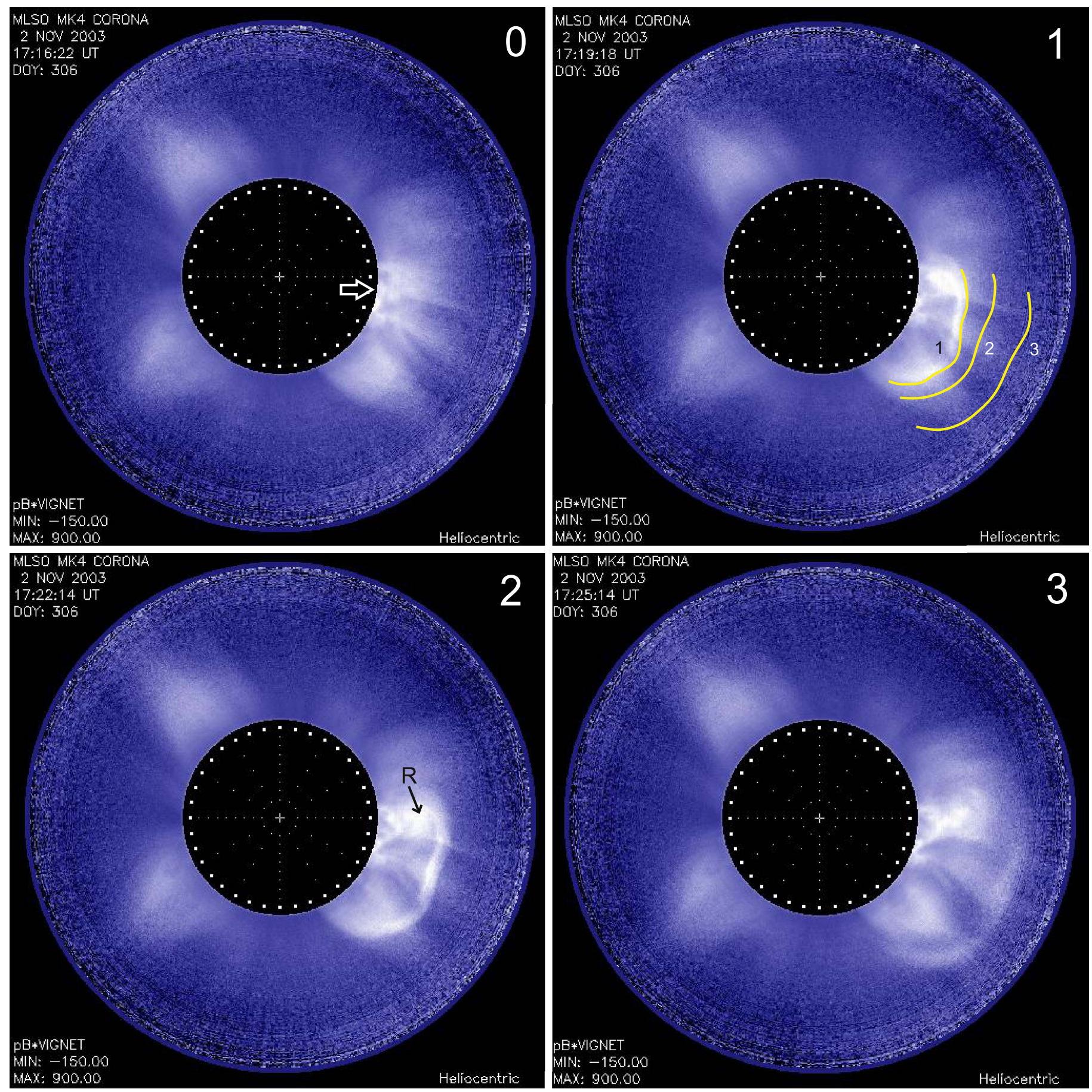

Figure 9. Evolution of the 2003 November 2 CME prior 17:25 UT: low corona images (MLSO Mk4), including a bright feature that disappears after the CME launch (arrow in panel 0), a rope-like structure (R), and the leading front (yellow contours, drawn from corresponding difference images). The CME's wide, bright front first appears at $1.7 R_{\odot}$ and then accelerates in the radial direction, keeping a constant angular extent until 17:25 UT.

While no imaging of the low-frequency type III sources is available for GLE-producing eruptions, there were observations of other events. Démoulin et al. (2012) reported several radio sources on the CME edges and concluded that those radio sources corresponded to energetic electrons accelerated during magnetic-reconnection processes between the CME and the ambient magnetic field. Morosan et al. (2014) have shown the low-frequency type III bursts at the expanding flank of a CME. Krupar et al. (2016) analyzed the type II and type III bursts and concluded that the radio emission arises from the flanks of the CME. Electrons generating type III bursts are produced at magnetic reconnection with open, nearly radial magnetic field lines, along which the electrons propagate.

It seems therefore plausible that emission of relativistic protons in the considered events starts with opening of largescale loops at the CME-driven reconnection with open magnetic field lines, most likely at the CME flank. The idea of the sudden release of previously accelerated and trapped particles is strongly supported by the observation that emissions of different particle species and energies rose simultaneously at the Sun, with a minute accuracy, as is evident from Figure 4 (also Kocharov et al. 2015, Figure 2(a) therein). An alternative explanation 


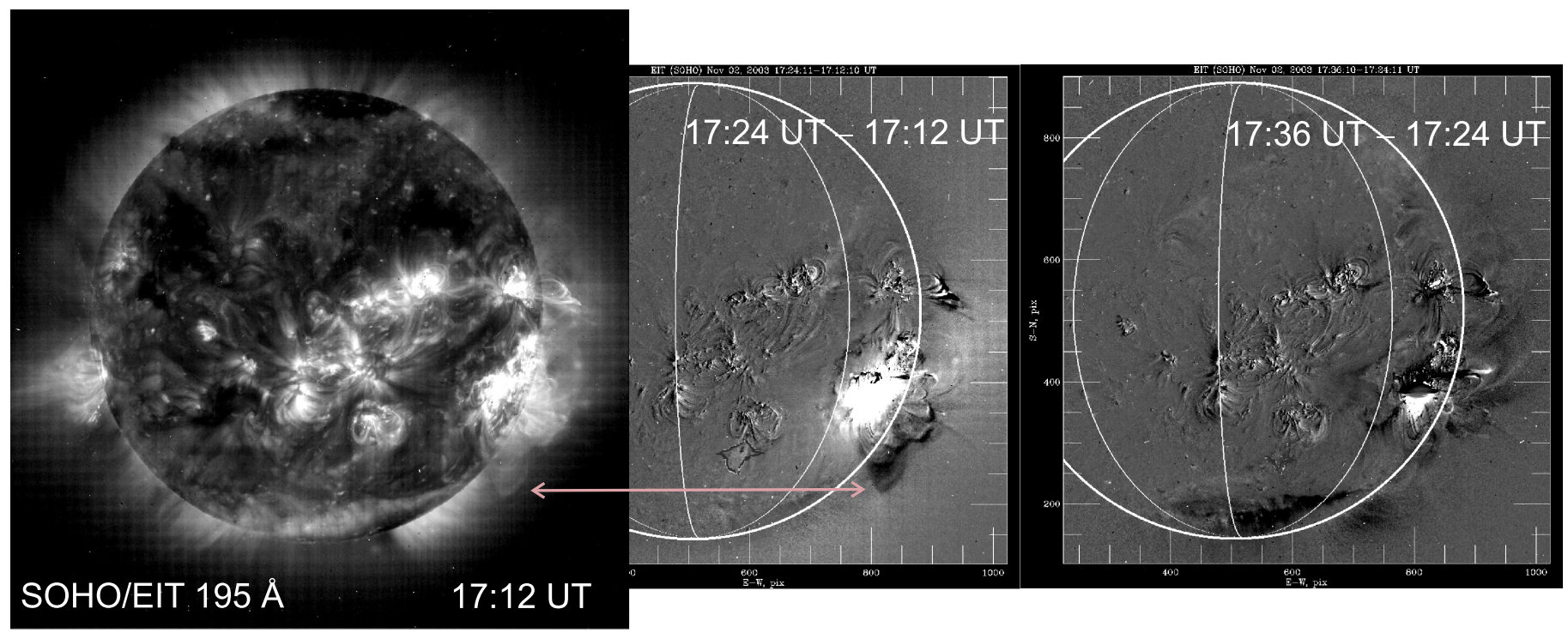

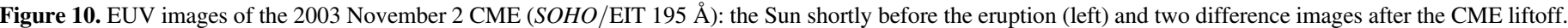

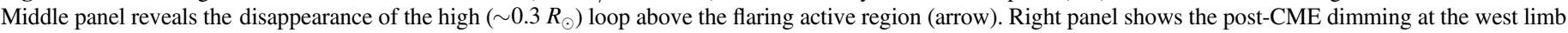
and around the south pole.

would be the particle acceleration to relativistic energies in one minute time, far from the flare in both space and time, which seems unlikely.

\subsection{Interplanetary Particles versus Interacting Particles in Other Events}

Parameters and history of the ion acceleration and trapping in the closed magnetic field structures of solar corona can be learned with data of their neutral secondary emissions, $\gamma$-rays, and neutrons. By comparing the inferred parameters of highenergy ions interacting at the Sun with parameters of ions escaping into the interplanetary space, it was concluded that for deka-MeV protons, the ratio of the numbers of interplanetary to interacting particles in gradual solar flares is often of the order of unity and the proton spectra for the interacting particles appear to be steeper than the corresponding spectra in the interplanetary space (Hua \& Lingenfelter 1987; Ramaty et al. 1993).

In the highest energy range of solar particles, there were detailed studies of the well-observed event of 1990 May 24 (GLE 48; Kocharov et al. 1994, 1996a, 1996b; Debrunner et al. 1997, and references therein). The main part of the GLE was caused by solar protons, rising at the Sun nearly 15 minutes after the flare impulsive phase, and the anisotropic flux of interplanetary protons continued for about 25 minutes (the prompt component of GLE). The interacting proton spectrum was deduced with data of the flare $\gamma$-ray emission, the solar highenergy neutrons (responsible for a part of the GLE), and the neutron-decay protons. Two components of interacting protons were produced in the flare impulsive phase: the first, softspectrum component and the second, hard-spectrum component. The total number of $>600 \mathrm{MeV}$ protons of the second interacting component was of the order of magnitude of the total number of the prompt component protons in space (Kocharov et al. 1996b). In the energy range of $100-1000 \mathrm{MeV}$, the spectrum of the second component of interacting protons was similar to or slightly softer than the spectrum of the prompt component protons emitted into the solar wind. Based on that observation, it was proposed that flare-accelerated protons were trapped in high, $0.3 R_{\odot}-0.6 R_{\odot}$ loops and then released into the interplanetary space after the loop opening (Kocharov et al. 1996a). Note that the $\gamma$-ray data of the 1990 May 24 flare indicated also the third production of interacting relativistic particles, a few minutes after the end of the flare impulsive phase and immediately after the Moreton wave transit (at around 20:51 UT; Kocharov et al. 1994, 1996b, respectively, Figures 5 and 1). In view of our present findings, we speculate that such postimpulsive-phase acceleration could be attributed to the CME launch.

\subsection{Common Scenario for the 1998 May 2 and 2003 November 2 Events}

Solar flares can accelerate particles even to relativistic energies, which is evident, in particular, from occasional observations of relativistic particles escaping into the interplanetary medium immediately after the flare impulsive phase (e.g., Klassen et al. 2005; Klein et al. 2014). Such prompt escape can be explained by the interchange reconnection between the closed field of the eruption core and open field nearby, which may occur within a minute after the flare pulse and allows the prompt access of the flare-accelerated particles from behind the CME to the solar wind (Masson et al. 2013). However, such prompt escape of the flare-accelerated relativistic protons from the Sun is not typically observed in GLEs (e.g., Gopalswamy et al. 2012). The CME launch in the two considered events was delayed with respect to the flare pulse for about 5 minutes, and the particle escape was delayed for another 5 minutes and more. The GLE delay can be explained by a delayed reconnection with the open field.

Two compositions, impulsive composition $(\mathrm{He} / \mathrm{p} \sim 0.1)$ and gradual composition $(\mathrm{He} / \mathrm{p} \sim 0.001-0.01)$, are observed in SEPs, suggesting two different sites of the particle energization. In the 1998 May 2 event, interplanetary ions in the energy range of $60-100 \mathrm{MeV}$ nucl $^{-1}$ were poor in helium, $\mathrm{He} / \mathrm{p} \sim 0.005$ (the SOHO/ERNE observation), which implies that they originated not from the flare but from another source of the particle energization, most likely from the CME liftoff. 

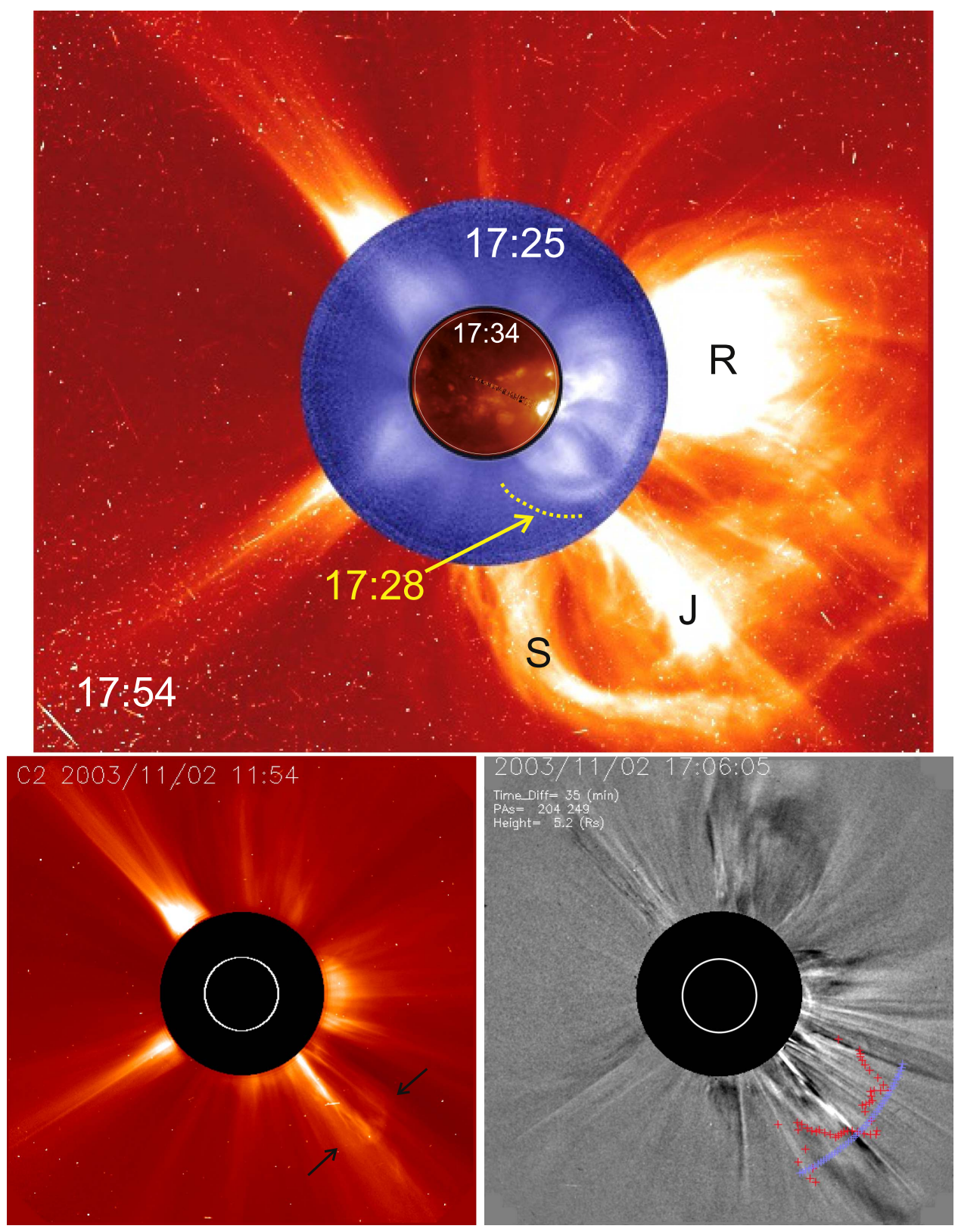

Figure 11. Evolution of the 2003 November 2 CME after 17:25 UT. Upper panel: images from center outward are obtained with GOES-12/SXI, MLSO Mk4, and LASCO C2. The yellow dotted line outlines the southern flank location in the MLSO difference image at 17:28 UT, indicative of the lateral expansion of CME starting at 17:25 UT and ending up with the transient coronal hole formation around the south pole (no later than 17:36 UT, Figure 10) and also with the flank brightening (S). The rope structure $(\mathrm{R})$ exists already in the low corona. The jet-like structure $(\mathrm{J})$ appears after 17:30 UT at the location of previous narrow, slow ejections along the streamer. Lower left: the previous CME imaged by LASCO C2 at 11:54 UT, first seen at 11:30 UT at position angle $224^{\circ}$ with width $33^{\circ}$ and speed $826 \mathrm{~km} \mathrm{~s}{ }^{-1}$ (between the arrows). Lower right: one of several subsequent ejections at the same location, shown with the difference image at 17:06 UT (GMU SEEDS).

Our findings are summarized in Figure 12. Impulsive phases of both flares comprise two pulses, $F_{1}$ and $F_{2}$. The sources $F_{1}$ and $F_{2}$ are situated at close but still different locations (1998 May 2, NRH), both are rich in hard X-rays as compared to microwaves (2003 November 2, RHESSI). The first pulse, $\mathrm{F}_{1}$, triggers the Moreton wave that either causes or just visualizes the eruption widening. After the flare pulse $F_{1}$, both eruptions develop in a similar way but with different rates (Figure 3). Note that Howard \& Pizzo (2016) recently presented statistical evidence that large-scale coronal eruptions associated with EIT waves exhibit characteristics that are consistent with a blast wave originating from a localized region (such as a solar flare). There is theoretical evidence that, in fast CMEs, flare reconnection is the primary mechanism responsible for both flare and CME (Karpen et al. 2012).

After the second pulse, $\mathrm{F}_{2}$, the soft X-ray flare has reached its maximum and further develops as a gradual flare. At the time of the soft X-ray maximum, the microwave-rich burst $\mathrm{C}_{1}$ starts to rise. The $\mathrm{C}_{1}$ source at $432 \mathrm{MHz}$ is situated beneath the $\mathrm{CME}$ and outside of the flaring active region (1998 May 2, Figure 6). While the microwave images are not available, we apply the identification $\mathrm{C}_{1}$ also to the source of the $1 \mathrm{GHz}$ emission, because of the observed joint development of emissions in the 400-1200 MHz frequency range (the PHOENIX-2 observation), even though their sources may be located at somewhat different altitudes. The $\mathrm{C}_{1}$ burst is relatively poor in hard 


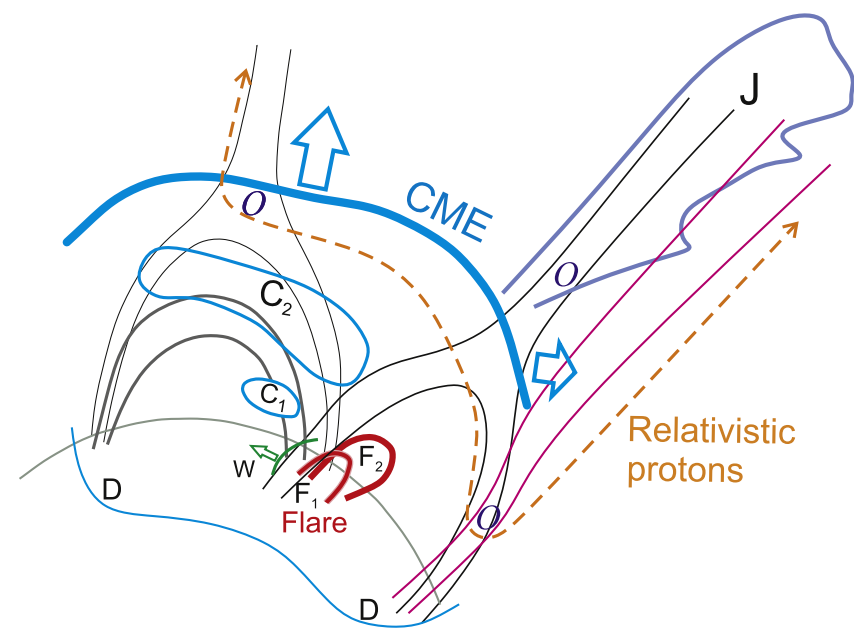

Figure 12. Eruption scenario for the two considered flare-CME-GLE events. The impulsive phase of the solar flare comprises two outbursts, $F_{1}$ and $F_{2}$. The first flaring causes the global coronal wave (W). The radio burst $C_{1}$ is associated with a CME-launch event, which may be triggered by the wave. The EUV dimming (D) and the extended radio source $\left(\mathrm{C}_{2}\right)$ are produced by the rising $\mathrm{CME}$ at different coronal altitudes. Injection of relativistic protons into the solar wind occurs upon the CME arrival at the top sections of helmet streamers and concurrently with the lateral expansion of CME at its right flank. Interaction $(\mathrm{J})$ between the $\mathrm{CME}$ loops originating from the flare region and the streamer is responsible for the second, more prolonged, emission of relativistic ions. Relativistic protons escape into the interplanetary medium at the CMEdriven magnetic reconnection with an open field $(O)$.

X-rays, as compared to the flare bursts $F_{1}$ and $F_{2}$ (Figure 8(a)), and its microwave spectrum does not extend to such high frequencies as the $F_{1}$ and $F_{2}$ spectra do (Figures 6 and 8(a)). A wide $\mathrm{CME}$ appears already at the heliocentric distance $\approx 1.7 R_{\odot}$ and then accelerates in the radial direction (Figure 9). The $\mathrm{C}_{1}$ burst coincides with the main acceleration period of the wide CME (Figure 8(a)). Therefore, the compact burst $C_{1}$ is regarded as a signature of the launch of the wide CME located above it, a part of the CME launch system.

The global radio source $\mathrm{C}_{2}$ is a kind of footprint of the entire CME. Assuming similar height-time profiles of the two eruptions (Figure 3), we estimate for the 1998 May $2 \mathrm{CME}$ that the $\mathrm{C}_{2}$-emission at $236-164 \mathrm{MHz}$ is produced during the CME transit through the upper sections of closed coronal structures situated at $1.8 R_{\odot}-2.3 R_{\odot}$. On 2003 November 2, when the $\mathrm{CME}$ nose reaches $2.3 R_{\odot}$, the $\mathrm{CME}$ also starts a lateral expansion at its southern flank (Figure 11). Concurrently, a late series of low-frequency type III radio bursts is produced (Figure 8(b)), which indicates magnetic reconnection with open magnetic field lines below the CME nose. First, emission of relativistic protons occurs simultaneously with those type III bursts. The accelerated particles are released from the largest loop-like magnetic structures after the CME-driven magnetic reconnection with open magnetic field at the CME flank and/or at tips of helmet streamers.

The proton injection profile of the 2003 November 2 event also comprises a less intensive injection after the first pulse, again in association with type III bursts (Figure 8(b), 17:30-17:48 UT). We speculate that those protons are released at magnetic reconnection between the CME-loops originating from the flare region (CME core) and the southwest streamer, as visualized by the brightening $(\mathrm{J})$.

While the $\gamma$-ray data are not available for the two considered events, observations of the 1990 May 24 flare allow us to suggest that the high-energy protons and helium are produced in the flare, then get trapped in the CME core for about 15 minutes, and finally escape after magnetic reconnection between the CME core and open field. Additionally, a helium-poor component shall be produced, most likely at the CME launch, a few minutes after the flare. The helium-poor component is trapped in the large-scale loops and then released by the expanding CME (the first and the only component recognized in the 1998 May 2 event near the Earth).

Our analysis of the 2003 November 2 GLE indicates that during 17:30-18:00 UT, the rigidity spectrum of protons steepens with rigidity but gradually evolves in time toward a single power law. This may be explained by the particle re-acceleration in large-scale magnetic loops and/or mixing of two components. The observed delays between the flare, the CME launch, and the GLE make it possible for the particles, initially accelerated at the flare and CME launch, to be further re-accelerated during the trapping in coronal and CME loops (for some models applicable to the re-acceleration in loops, see Kocharov et al. 2012; Afanasiev et al. 2014).

\section{Conclusions}

We have analyzed the data of particle and electromagnetic observations of the two GLE-producing flare-CME events, events of 1998 May 2 and 2003 November 2. For the first time, we have deduced the time profile of the relativistic proton source at the Sun and compared it with data of electromagnetic observations including both the dynamic spectra and images. Based on the multi-wavelength analysis of the two eruptions, and additionally taking into account the data of high-energy observations of the 1990 May 24 event, we conclude the following.

1. Inspection of global morphology of involved active regions, magnetic field structures, and CMEs reveals a qualitative similarity between the two eruptions, while the eruptions are strongly distinct in total energy. Time profiles of the soft X-ray flare, the CME height, and the relativistic proton emission of one eruption coincide with corresponding profiles of another eruption after a rescaling of time according to the CME speed, $V_{\mathrm{CME}}$, with the flare's first major pulse being the eruption triggering event, and the soft X-ray flare magnitude scaled as a power of the CME speed: $I_{\mathrm{X}} \sim V_{\mathrm{CME}}^{3.3}$.

2. A common scenario is possible for both GLE-producing eruptions. Two major pulses of the flare's impulsive phase, $F_{1}$ and $F_{2}$, are strong in both hard X-rays and microwaves. The first pulse causes a global coronal wave. The wave could trigger, in particular, a compact, microwave-rich burst $C_{1}$ situated outside the flare active region. The $C_{1}$ burst coincides with the main acceleration period of CME in the low corona. We identify the decimetric-continuum burst $\mathrm{C}_{1}$ with a $\mathrm{CME}$ launch event.

3. Particle transport from Sun to Earth in the 2003 November 2 event was essentially not free of scattering and, therefore, the transport modeling shall be applied to deduce the time profile of the relativistic proton injection at the Sun. The inferred solar source of the 2003 November 2 GLE exhibits a strong, $\approx 5$ minute long pulse followed by $\mathrm{a} \approx 20$ minute long period of a less intensive proton emission.

4. First emission of relativistic protons from the Sun begins when CME reaches the tips of helmet streamers. In the 
2003 November 2 event, the first pulse of the proton emission also coincides with a well-observed period of fast lateral expansion of CME. The second period of the proton emission may be ascribed to the interaction of the CME core with the coronal streamer. The proton emissions coincide with a series of the low-frequency type III radio bursts, indicative of the magnetic reconnection with open magnetic field lines, driven by the rising CME.

5. Observations support the idea that in the two considered events, relativistic protons originate from the large-scale magnetic traps opened up by the CME-driven magnetic reconnection.

We find it plausible that solar high-energy particles are initially accelerated during the flare and the CME launch. The particle acceleration associated with the CME launch in solar corona may be responsible for the helium-poor component of SEPs that is often ascribed to the acceleration in the CME-bow shock in solar wind. The particles accelerated in the beginning of the two considered flare-CME events then populate large, $\sim R_{\odot}$ magnetic loops, and could be re-accelerated there. Some protons may remain trapped in the large-scale loops for a long time and eventually precipitate into the chromosphere to produce the high-energy secondary emissions, like pion-decay $\gamma$-rays, and neutrons, at different locations on the Sun, which may be visible even for behind-the-limb flares (as observed by Ackermann et al. 2017). However, a significant fraction of the trapped particles escape to the solar wind at the CME-induced magnetic field opening. The typically observed delay between the flare impulsive phase and the injection of solar relativistic protons into the interplanetary medium does not contradict their flare origin because the delay is caused by the coronal trapping.

This research was supported by the Academy of Finland through projects 260596, 258963, and 272157. A.K. was supported by the German DLR under grant 50 OC 1302. J.L. was supported by the BK21 Plus Program (21A20131111123) funded by the Ministry of Education (MOE, Korea) and National Research Foundation of Korea (NRF). M.K. acknowledges support from Grant P209/12/0103 of the Grant Agency of the Czech Republic. SOHO is a project of international cooperation between ESA and NASA.

\section{Appendix A \\ Interplanetary Magnetic Field on 2003 November 2}

The state of the IMF is important for the particle transport to $1 \mathrm{au}$. The IMF data are available from the $A C E$ spacecraft orbiting near the Lagrange point L1. At the observed solar wind speed of $U \approx 520 \mathrm{~km} \mathrm{~s}^{-1}$, a time shift of $\approx-48$ minutes is implied between the plasma parcel at L1 and the same parcel near the Earth's orbit. The IMF data are plotted in Figure 13 versus the $A C E$ time, while the GLE timing (bars 1 and 2) is shifted back in time to fit with the solar wind timing on $A C E$.

In the present work, we analyze in detail only the first period of GLE 67, shown with the red bar. During that period, the IMF direction changes irregularly, with the amplitude of $\approx 15^{\circ}$, at the timescales corresponding to the Larmor radius of $\approx 1.5 \mathrm{GV}$ proton, $\Delta t=R_{\mathrm{L}} / U \approx 28$ minutes. The lower panel of Figure 13 shows that in the first period of the particle event, the magnetic instrument moves with respect to plasma across the magnetic field lines at the angle of $\sim 70^{\circ}$. Hence, the
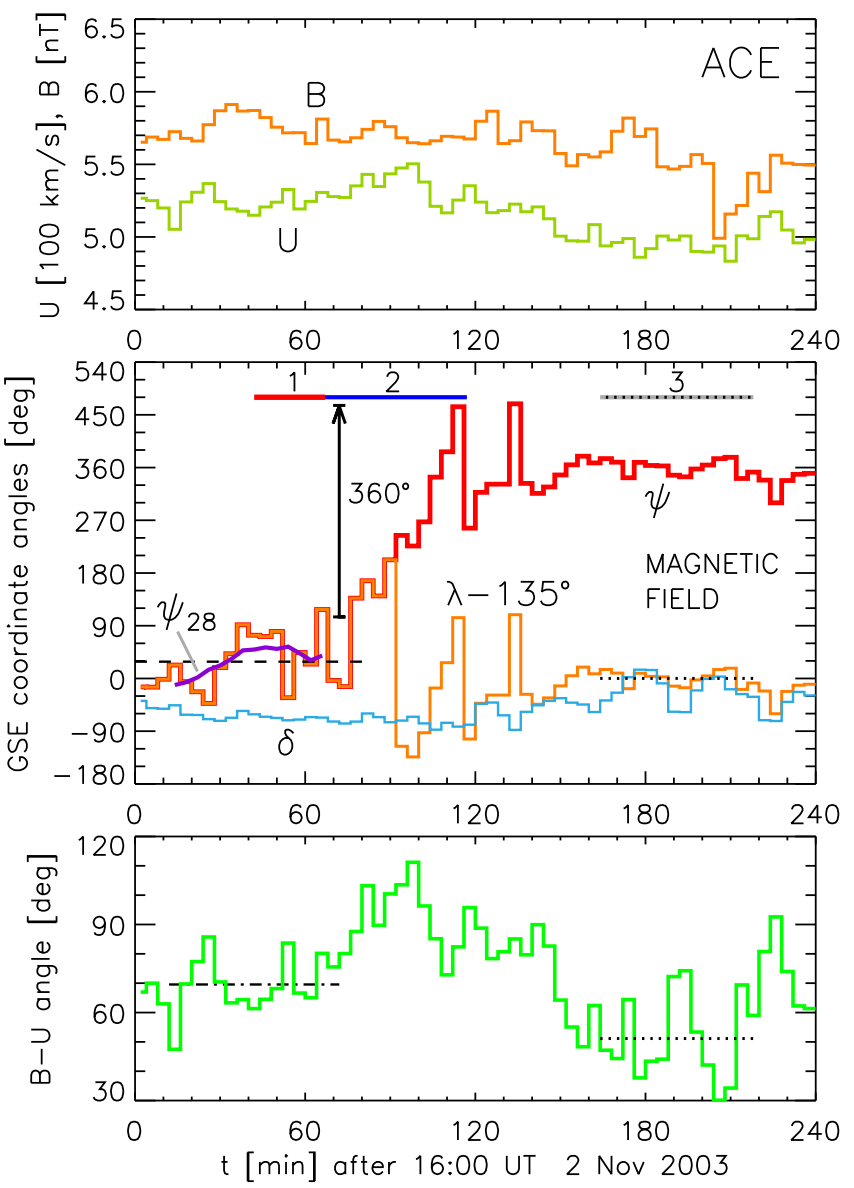

Figure 13. Solar wind velocity and magnetic field observed on $A C E$. Upper panel: the 4-minute average magnetic field intensity, $B$, and plasma speed, $U$. Middle panel: the magnetic field angles in the GSE coordinate system, $\lambda$ and $\delta$. The azimuthal angle $\lambda$ is shown either as counted from the nominal IMF direction, $\lambda-135^{\circ}$ (orange histogram) or as the cumulative rotation angle $\psi$, counted from the same, nominal IMF direction (red histogram). The angle $\psi_{28}$ is the gliding 28-minute average of $\psi$, while the dashed line indicates the 80 minute average value $\langle\psi\rangle_{80}$. The difference $\psi_{28}-\langle\psi\rangle_{80}$ illustrates the magnitude of fluctuations at the proton Larmor radius scale. Red and blue horizontal bars, 1 and 2, indicate two periods of the GLE, shifted by -48 minutes to account for the distance between the particle detectors on the Earth and the magnetometer at the L1 point. Lower panel: the angle between magnetic field and plasma velocity.

observed variance in the magnetic field direction is largely due to the magnetic shear.

Note that the second period of the proton event (blue bar) is observed inside a distinct magnetic tube, because the magnetic field vector makes a full rotation around the south-north axis, staying $\approx 30^{\circ}$ off the axis. Because the GLE's period 2 exactly coincides with the passage of the distinct tube, any changes in the particle flux at the start and the end of period 2 may be spatial in nature. As a reference, we also show a period of nearly standard IMF, observed later during the time interval indicated with bar 3 .

\section{Appendix B \\ Modeling the Response of Neutron Monitor Network}

Here we describe a fitting procedure applied by A. Mishev to the data of the neutron monitor network of the 2003 November 2 event (also Mishev et al. 2014). This fitting procedure does not consider interplanetary transport but allows for a search over a very wide parameter region, and hence can be used at 

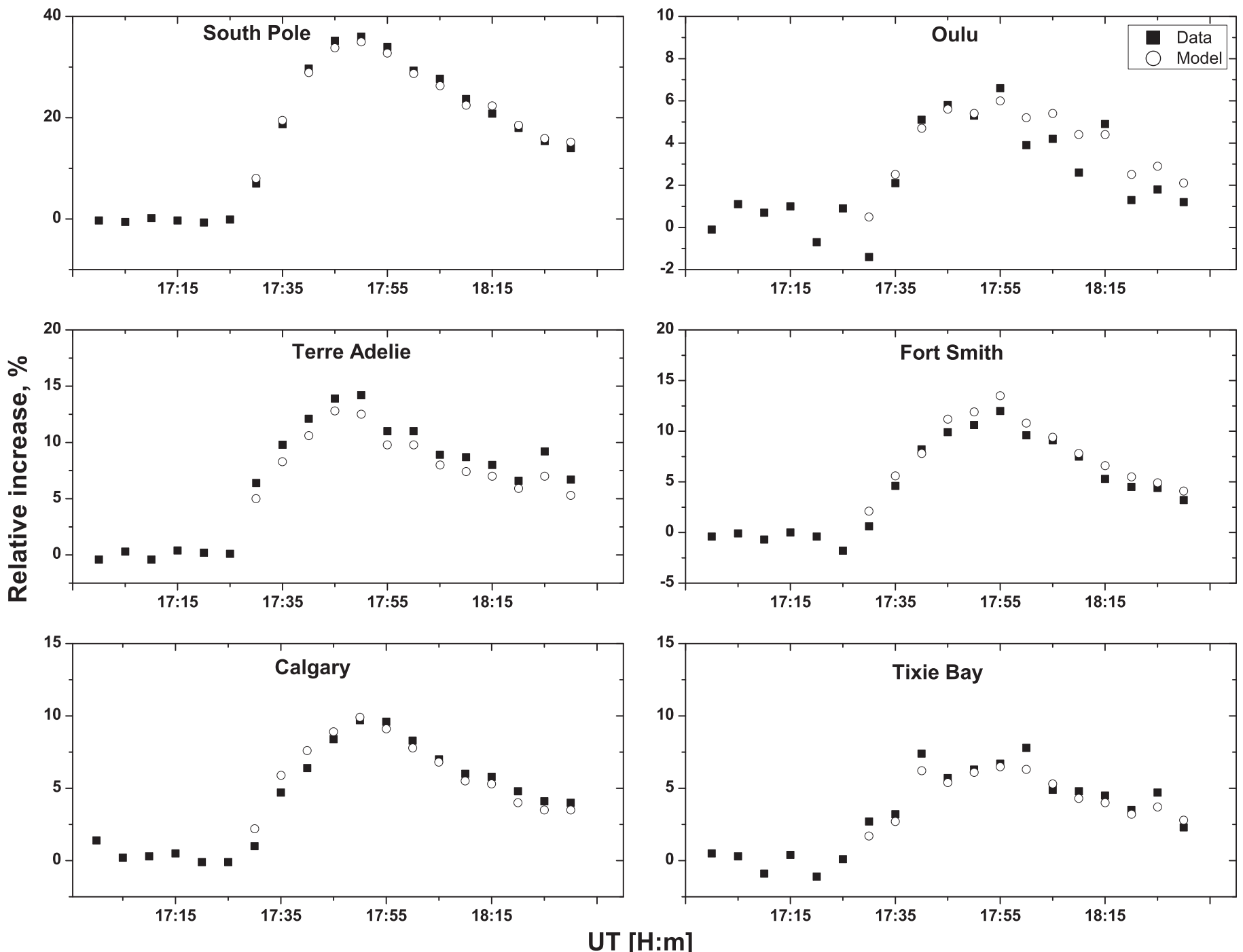

Figure 14. Modeled and observed responses of six NM stations during the 2003 November 2 event. The quality of the modeled responses for other stations is of the same order.

the first stage of the GLE fitting. The second stage of the fitting procedure will be described in Appendix C. It will include also the interplanetary transport modeling, while the parameter region will be strongly limited based on the results of stage one.

The relative count rate increase of a given neutron monitor (NM) caused by solar high-energy protons and helium is of the form

$$
\frac{\Delta N}{N}=\frac{\int_{P_{\text {cut }}}^{P_{\max }} J_{\| \mathrm{SEP}}(P) G(\alpha(P)) Y(P) d P}{\int_{P_{\text {cut }}}^{\infty} J_{\mathrm{GCR}}(P) Y(P) d P},
$$

where $P$ is rigidity of proton or $\alpha$-particle; $J_{\| \operatorname{SEP}}(P)$ is the rigidity spectrum of solar particles in the direction of the maximal flux; $G(\alpha(P))$ is the pitch angle distribution of solar particles; the pitch angle $\alpha$ is defined as the angle between the direction of particle motion outside the magnetosphere (asymptotic direction) and the axis of particle flux anisotropy; the function $\alpha(P)$ is determined by particle trajectories through the magnetosphere to a neutron monitor on the ground; $Y(P)$ is the NM yield function; $J_{\mathrm{GCR}}(P)$ is the rigidity spectrum of galactic cosmic-rays (GCR); $N$ is the background count rate of the galactic origin; $\Delta N$ is the count rate increase due to solar particles; $P_{\text {cut }}$ is the minimum (cut-off) rigidity of the station and $P_{\max }$ is the maximum rigidity of the solar particle spectrum adopted here to be $20 \mathrm{GV}$.

We model the solar particle spectrum as a modified power law in rigidity:

$$
J_{\|}(P)=J_{0} P^{-\gamma+\delta \gamma[1-(P / 1 \mathrm{GV})]},
$$

where $J_{\|}$is the particle flux arriving from the Sun along the axis of symmetry whose direction is defined by geographic coordinate angles $\Psi$ and $\Lambda$ (latitude and longitude), $\gamma$ is the power-law spectral exponent at a rigidity of $P=1 \mathrm{GV}$, and $\delta \gamma$ is the rate of the spectrum steepening. The pitch angle distribution is modeled as a superposition of two Gaussians:

$$
G(\alpha) \sim \exp \left(-\alpha^{2} / \sigma_{1}^{2}\right)+C \exp \left(-(\alpha-\pi)^{2} / \sigma_{2}^{2}\right),
$$

where $\sigma_{1(2)}$ is the distribution width. With parameter $C \neq 0$, we can account for additional particle flux from the anti-Sun 

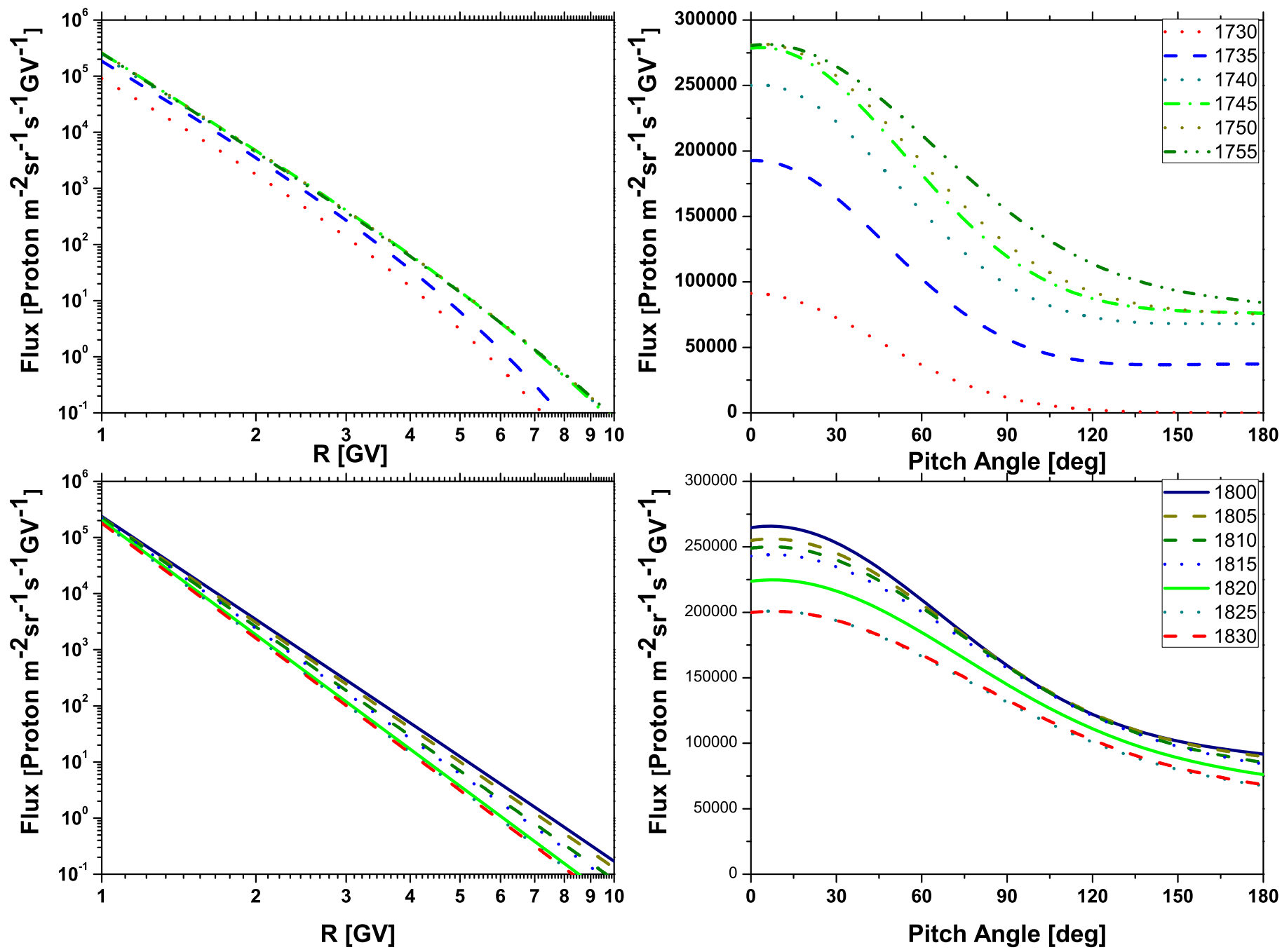

Figure 15. Derived rigidity spectra and pitch angle distributions of solar relativistic ions during the 2003 November 2 GLE. The spectra are for the flux arriving from the Sun along the symmetry axis. Time denoted in the legend refers to the beginning of the corresponding 5-minute interval.

direction, if required by observational data. Thus, eight parameters have to be determined: $J_{0}, \gamma, \delta \gamma, \Psi, \Lambda, \sigma_{1}, \sigma_{2}$, and $C$.

The solution of the inverse problem is based on the Levenberg-Marquardt method (Levenberg 1944; Marquardt 1963), where the optimization is performed by the minimization of the sum of squared differences between the modeled NM responses and the observed NM responses, i.e., optimization of the functional $\mathcal{F}$ over the vector of unknowns for the total $m$ neutron monitor stations for a particular time bin,

$$
\mathcal{F}=\sum_{i=1}^{m}\left[\left(\frac{\Delta N_{i}}{N_{i}}\right)_{\text {mod }}-\left(\frac{\Delta N_{i}}{N_{i}}\right)_{\text {obs }}\right]^{2} .
$$

We use a general quality criterium for a goodness of the fit (e.g., Himmelblau 1972):

$$
\mathcal{D}=\frac{\sqrt{\sum_{i=1}^{m}\left[\left(\frac{\Delta N_{i}}{N_{i}}\right)_{\bmod }-\left(\frac{\Delta N_{i}}{N_{i}}\right)_{\mathrm{obs}}\right]^{2}}}{\sum_{i=1}^{m}\left(\frac{\Delta N_{i}}{N_{i}}\right)_{\mathrm{obs}}} .
$$

In the case of a moderately strong event like GLE 67, we could accept any parameter set that provides the total deviation $\mathcal{D}<8 \%$ (at 5-minute time bins), because of unavoidable statistical fluctuations. However, we additionally consider separately every station and compare the modeled increase with the observed one. Based on such a consideration, we reject all cases with disagreement exceeding $10 \%$ for any station. Besides, we require that the total number of stations with underestimated signals is nearly equal to the number of stations with overestimated signals. The best fit is one that minimizes $\mathcal{D}$ at the additional conditions.

For the analysis of GLE 67, 27 NM stations were jointly considered. Examples of the NM count rate fits are shown in Figure 14. Figure 15 shows the best-fit rigidity spectra obtained for various periods of the event and the corresponding pitch angle distributions. Deduced pitch angle distributions are rather wide, even before the maximum of the event, during 17:35-17:45 UT, the angular width $\sigma_{1}^{2} \approx 1.5 \mathrm{rad}^{2}$.

In an additional round of calculations, we have fitted the first period of GLE, 17:30-18:10 UT with an exponential rigidity spectrum. Best-fit spectra of both spectrum models, exponential and modified power laws, come close to each other in the rigidity range of $1.5-6 \mathrm{GV}$, implying that this is the efficient proton energy range for the GLE production (the range of $1.4-4.4 \mathrm{GeV}$ is adopted for plotting the theoretical intensity profile in Figure 5). 


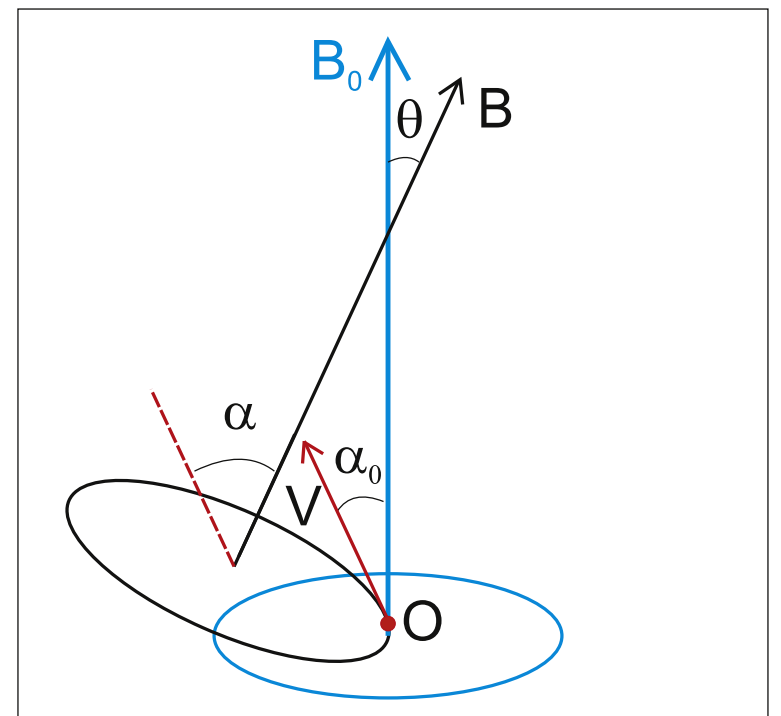

Figure 16. Guiding magnetic field with a random shear $(\theta)$. Particle velocity $\boldsymbol{V}$ makes the pitch angle $\alpha$ with magnetic field at the Larmor circle center and a different angle, $\alpha_{0}$, with magnetic field at the observation point $(\mathrm{O})$.

\section{Appendix C}

Interplanetary Transport Model for the 2003 November 2 Event

SEPs propagating in the magnetic field of the solar wind experience two concurrent, competing processes: magnetic focusing, due to the overall decrease in the IMF intensity, and scattering at small-scale magnetic irregularities/waves-both are included in Equation (1). The Boltzmann equation can be replaced/solved with a stochastic simulation of the particle transport (e.g., Kocharov et al. 1998, Appendix B therein). Our stochastic simulations account for all processes included in Equation (1) and for two additional effects (see below). The pitch angle diffusion is modeled at $q=5 / 3$, the Kolmogorov turbulence spectrum. The proton mean-free path for the smallangle scattering, $\lambda$, is independent of the distance within $0.1 \mathrm{au}$ from the Sun and then increases linearly with the field-aligned coordinate (the $\lambda$ values given in the figures are for $1 \mathrm{GeV}$ proton near the Earth orbit). The large-scale IMF is according to the standard, Parker's model. However, we additionally compare the standard modeling results with the case of radial diffusion, to estimate an effect of the IMF model on the inferred source profile at the Sun.

Data of the neutron monitor network indicate that along with the particle flux from the Sun, there is an isotropic component present from the very beginning of the event. Such a pattern can be reproduced by adding to the model a random isotropization process (like the SSI scattering by Kocharov et al. 1998). We propose that in the strongly disturbed magnetic environment of 2003 November 2, some irregularities in IMF were abrupt enough to scatter protons over a large angle. The large-angle scattering is modeled as a random, small-chance isotropization with corresponding mean-free path $\lambda_{\mathrm{SSI}}=0.3 \mathrm{au}$, at all proton locations and energies (excluding protons propagating nearly parallel to the magnetic field: $|\mu|>0.9$ ).

In the first period of the 2003 November 2 event, the magnetic field orientation exhibits a strong variance along the perpendicular to IMF direction (the $A C E$ observation; Appendix A), which looks like a random magnetic shear. The random magnetic shear introduces an uncertainty to the guiding field

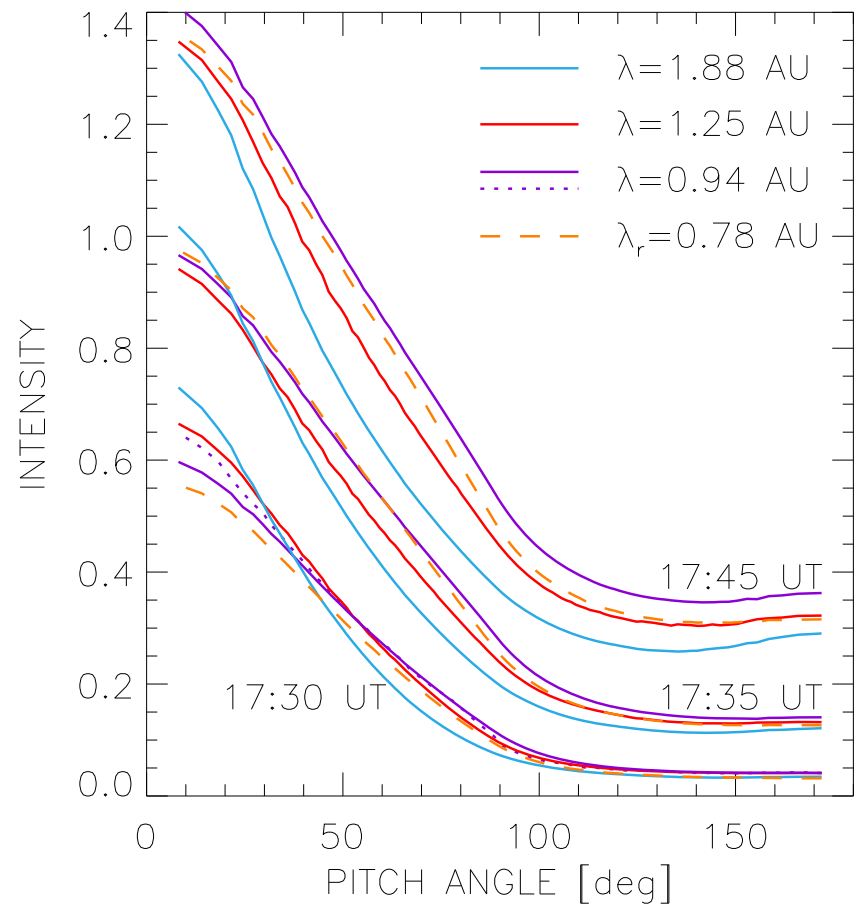

Figure 17. Model pitch angle distributions for three 5-minute bins of the first period of GLE 67. Shown are three cases of focused transport in the standard IMF of Parker's solar wind, $\lambda=0.94 \mathrm{au}, 1.25 \mathrm{au}$, and $1.88 \mathrm{au}$, and one case of the radial diffusion, $\lambda_{r}=0.78 \mathrm{au}$. The middle case, $\lambda=1.25 \mathrm{au}$, provides a better fit to the observed NM count rates than two other cases. However, $\lambda=0.94$ au and 1.88 au are still acceptable. Corresponding source profiles for the standard IMF are shown in the lower panel of Figure 8(b). For the radial diffusion case, the source is identical to the source of the standard model with $\lambda=1.88 \mathrm{au}$. For a comparison, we show with the dotted line one distribution not convolved with the mixing matrix $P\left(\mu, \mu_{0}\right)$. It is seen that the effect of mixing is of the order of observational uncertainties for this GLE.

direction and hence, to the particle pitch-angle (Figure 16). The effect of such uncertainty on the "observed" pitch-angle distribution is simulated by mixing the model pitch-angle distributions corresponding to different guiding field orientations. We randomize the guiding field direction in the already simulated pitch angle distribution of protons at 1 au. We propose that the guiding field direction $\boldsymbol{B}$ experiences random fluctuations around the average direction $\boldsymbol{B}_{0}$, so that the angle $\theta$ between the vectors possesses a normal distribution with a standard deviation $\sigma_{B}$ (based on the IMF measurements, we adopt for the 2003 November 2 event the value $\sigma_{B}=15^{\circ}$ ). The cosine of the angle between the particle velocity $\boldsymbol{V}$ and the average magnetic field direction $\boldsymbol{B}_{0}$ is

$$
\mu_{0}=\mu \cos \theta+\sqrt{1-\mu^{2}} \sin \theta \sin \left(\omega_{B} t\right)
$$

$\mu_{0}=\cos \alpha_{0}, \mu=\cos \alpha$, and $\omega_{B}$ is the angular frequency of proton gyration. By randomization of the magnetic field angle $\theta$ and the particle phase angle $\omega_{B} t$, we find a probability that a particle of the pitch angle cosine $\mu$ makes with the average magnetic field direction $\boldsymbol{B}_{0}$ the pitch angle of cosine $\mu_{0}$, $P\left(\mu, \mu_{o}\right)$. The mixing matrix $P\left(\mu, \mu_{o}\right)$ is convolved with the originally simulated pitch-angle distribution to get the final pitch angle distribution to be compared with observational data.

Figure 17 shows the model pitch angle distributions consistent with the neutron monitor data. The comparison with NM data is done with use of the quality criterium $\mathcal{D}$ defined in Appendix B. While the range of acceptable values of the mean- 
free path is relatively large, $\lambda=0.94-1.88 \mathrm{au}$, it is translated into only 1-2 minute uncertainty in the proton source profiles at the Sun (Figure 8(b)), because the proton speed is very high.

As an extreme alternative to the standard transport model, we consider a radial transport that would be a radial diffusion, if the mean-free path was somewhat smaller. In the alternative model $\left(\lambda_{r}=0.78\right.$ au in Figure 17), we simulate particle scattering and focusing in the radial magnetic field, with the same processes of small-angle scattering and large-angle scattering as in the standard model. It turns out, however, that the replacement of the standard transport model with the model of radial transport does not change the solar source profile much. This is because the inferred history of particle production depends mainly on the angular width of the proton flux distribution, which is equally well reproduced in both models. Recall that, in the standard focused transport model, the flux anisotropy axis is parallel to the magnetic field, while the radial diffusion suggests the anisotropy along the Sun-Earth line.

\section{References}

Ackermann, M., Allafort, A., Baldini, L., et al. 2017, ApJ, 835, 219 Adriani, O., Barbarino, G. C., Bazilevskaya, G. A., et al. 2011, ApJ, 742, 102 Afanasiev, A., Vainio, R., \& Kocharov, L. 2014, ApJ, 790, 36 Bougeret, J. L., Kaiser, M. L., Kellogg, P. J., et al. 1995, SSRv, 71, 231 Brueckner, G. E., Howard, R. A., Koomen, M. J., et al. 1995, SoPh, 162, 357 Cane, H. V., Erickson, W. C., \& Prestage, N. P. 2002, JGR, 107, 1315 Clem, J., \& Dorman, L. 2000, SSRv, 93, 335

Cliver, E. W. 1996, in AIP Conf. Proc. 374, High Energy Solar Physics, ed. R. Ramaty, N. Mandzhavidze, \& X.-M. Hua (New York: Woodbury), 45 Cramp, J. L., Duldig, M. L., Flückiger, E. O., et al. 1997, JGR, 102, 24237 Cramp, J. L., Humble, J. E., \& Duldig, M. L. 1995, PASAu, 11, 28 Dalla, S., Marsh, M. S., Kelly, J., \& Laitinen, T. 2013, JGR, 118, 5979 Danilova, O. A., Tyasto, M. I., Vashenyuk, E. V., et al. 1999, in Proc. 26th Int. Cosmic Ray Conf. 6, ed. D. Kieda, M. Salamon, \& B. Dingus (IUPAP: Salt Lake City), 399

Debrunner, H., \& Brunberg, E. 1968, CaJPh, 46, 1069

Debrunner, H., \& Lockwood, J. A. 1980, JGR, 85, 6853

Debrunner, H., Lockwood, J. A., Barat, C., et al. 1997, ApJ, 479, 997

Delaboudiniere, J.-P., Artzner, G. E., Brunaud, J., et al. 1995, SoPh, 162, 291 Démoulin, P., Vourlidas, A., Pick, M., \& Bouteille, A. 2012, ApJ, 750, 147

Desorgher, L., Kudela, K., Flückiger, E. O., et al. 2009, AcGeo, 57, 75

Dorman, L. I. 2004, Cosmic Rays in the Earth's Atmosphere and Underground (Dordrecht: Kluwer)

Dröge, W., Kartavykh, Y. Y., Klecker, B., \& Kovaltsov, G. A. 2010, ApJ, 709, 912

Earl, J. A. 1981, ApJ, 251, 739

Elmore, D. F., Burkepile, J. T., Darnell, J. A., Lecinski, A. R., \& Stanger, A. L. 2003, Proc. SPIE, 4843, 66

Gil, A., Usoskin, I. G., Kovaltsov, G. A., et al. 2015, JGR, 120, 7172

Gopalswamy, N., Xie, H., Yashiro, S., et al. 2012, SSRv, 171, 23

Gopalswamy, N., Yashiro, S., Thakur, N., et al. 2016, ApJ, 833, 216

Hatton, C. 1971, in Progress in Elementary Particle and Cosmic-ray Physics X, ed. J. G. Wilson \& S. A. Wouthuysen (Amsterdam: North Holland Publishing Co.), 1

Himmelblau, D. M. 1972, Applied Nonlinear Programming (New York: McGraw-Hill Book Company)

Howard, T. A., \& Pizzo, V. J. 2016, ApJ, 824, 92

Hua, X.-M., \& Lingenfelter, R. E. 1987, SoPh, 107, 351

Hurford, G. J., \& Gary, D. E. 1989, BAAS, 21, 861

Ipavich, F. M., Galvin, A. B., Lasley, S. E., et al. 1998, JGR, 103, 17205

Jiricka, K., Karlicky, M., Kepka, O., \& Tlamicha, A. 1993, SoPh, 147, 203

Karpen, J. T., Antiochos, S. K., \& DeVore, C. R. 2012, ApJ, 760, 81
Kerdraon, A., \& Delouis, J. M. 1997, in Coronal Physics from Radio and Space Observations, ed. G. Trottet (Berlin: Springer), 192

Klassen, A., Krucker, S., Kunow, H., et al. 2005, JGR, 110, A09S04

Klein, K.-L., Masson, S., Bouratzis, C., et al. 2014, A\&A, 572, A4

Kocharov, L., Klassen, A., Valtonen, E., Usoskin, I., \& Ryan, J. M. 2015, ApJL, 811, L9

Kocharov, L., Kovaltsov, G., Torsti, J., et al. 1996a, in AIP Conf. Proc. 374, High Energy Solar Physics, ed. R. Ramaty, N. Mandzhavidze, \& X.-M. Hua (New York: Woodbury), 246

Kocharov, L., Kovaltsov, G. A., Torsti, J., \& Huttunen-Heikinmaa, K. 2005, JGR, 110, A12S03

Kocharov, L., Reiner, M. J., Klassen, A., Thompson, B. J., \& Valtonen, E. 2010, ApJ, 725, 2262

Kocharov, L., Saloniemi, O., Torsti, J., et al. 2007, ApJ, 659, 780

Kocharov, L., Torsti, J., Vainio, R., Kovaltsov, G. A., \& Usoskin, I. G. 1996b, SoPh, 169, 181

Kocharov, L., Vainio, R., Kovaltsov, G. A., \& Torsti, J. 1998, SoPh, 182, 195

Kocharov, L., Vainio, R., Pomoell, J., et al. 2012, ApJ, 753, 87

Kocharov, L. G., Lee, J. W., Zirin, H., et al. 1994, SoPh, 155, 149

Krupar, V., Eastwood, J. P., Kruparova, O., et al. 2016, ApJL, 823, L5

Kunow, H., Wibberenz, G., Green, G., Müller-Mellin, R., \& Kallenrode, M.-B. 1991, in Physics of Inner Heliosphere, Vol. 2, ed. R. Schwenn \& E. Marsch (Berlin: Springer-Verlag), 262

Kunstmann, J. 1979, ApJ, 229, 812

Lee, J., Gary, D. E., \& Shibasaki, K. 2000, ApJ, 531, 1109

Levenberg, K. 1944, QApMa, 2, 164

Lin, R. P., Dennis, B. R., Hurford, G. J., et al. 2002, SoPh, 210, 3

Mann, G., Aurass, H., Voigt, W., \& Paschke, J. 1992, Coronal Streamers, Coronal Loops, and Coronal and Solar Wind Composition (ESA Special Publication), Vol. 348, ed. C. Mattok (Noordwijk: ESA), 129

Marquardt, D. 1963, SJAM, 2, 431

Masson, S., Antiochos, S. K., \& DeVore, C. R. 2013, ApJ, 771, 82

McComas, D. J. 1998, SSRv, 86, 563

Messmer, P., Benz, A. O., \& Monstein, C. 1999, SoPh, 187, 335

Mishev, A., Kocharov, L., \& Usoskin, I. 2014, JGR, 119, 670

Mishev, A., \& Usoskin, I. 2013, Journal of Physics: Conference Series, 409, 012152

Mishev, A., Usoskin, I., \& Kovaltsov, G. 2013, JGR, 118, 2783

Morosan, D. E., Gallagher, P. T., Zucca, P., et al. 2014, A\&A, 568, A67

Ogawara, Y., Takano, T., Kato, T., et al. 1991, SoPh, 136, 1

Olmedo, O., Zhang, J., Wechsler, H., Poland, A., \& Borne, K. 2008, SoPh, 248, 485

Parker, E. N. 1965, P\&SS, 13, 9

Pohjolainen, S., Maia, D., Pick, M., et al. 2001, ApJ, 556, 421

Ramaty, R., Mandzhavidze, N., Kozlovsky, B., \& Skibo, J. G. 1993, AdSpR, 13,275

Reiner, M. J., Klein, K.-L., Karlicky, M., et al. 2008, SoPh, 249, 337

Ruffolo, D., Tooprakai, P., Rujiwarodom, M., et al. 2006, ApJ, 639, 1186

Ryan, J. M., Lockwood, J. A., \& Debrunner, H. 2000, SSRv, 93, 35

Saito, K., Poland, A. I., \& Munro, R. H. 1977, SoPh, 55, 121

Schatten, K. H., Wilcox, J. M., \& Ness, N. F. 1969, SoPh, 6, 442

Scherrer, P. H., Bogart, R. S., Bush, R. I., et al. 1995, SoPh, 162, 129

Schrijver, C. J., \& DeRosa, M. L. 2003, SoPh, 212, 165

Shea, M. A., \& Smart, D. F. 1982, SSRv, 32, 251

Shea, M. A., \& Smart, D. F. 2012, SSRv, 171, 161

Smith, C. W. 1998, SSRv, 86, 613

Takahashi, T., Mizuno, Y., \& Shibata, K. 2016, ApJL, 833, L8

Thompson, B. J., Cliver, E. W., Nitta, N., Delanné, C., \& Delaboudinière, J.-P. 2000, GeoRL, 27, 1431

Ting, S. 2013, NuPhS, 243, 12

Toptygin, I. N. 1985, Cosmic Rays in Interplanetary Magnetic Fields (Dordrecht: Reidel)

Torsti, J., Riihonen, E., \& Kocharov, L. 2004, ApJL, 600, L83

Torsti, J., Valtonen, E., Lumme, M, et al. 1995, SoPh, 162, 505

Tsuneta, S., Acton, L., Bruner, M., et al. 1991, SoPh, 136, 37

Yashiro, S., Gopalswamy, N., Michalek, G., et al. 2004, JGR, 109, A07105

Zhang, M., Qin, G., \& Rassoul, H. 2009, ApJ, 692, 109 